\title{
Equation of state of fluid methane from first principles with machine learning potentials
}

\author{
Max Veit* and Gábor Csányi \\ Engineering Laboratory \\ University of Cambridge \\ Trumpington Street \\ Cambridge, CB2 1PZ \\ United Kingdom \\ Sandeep Kumar Jain, Satyanarayana Bonakala, and Indranil Rudra \\ Shell India Markets Pvt. Ltd. \\ Bengaluru 562149 \\ Karnataka, India \\ Detlef Hohl \\ Shell Global Solutions International BV \\ Grasweg 31 \\ $1031 \mathrm{HW}$ Amsterdam \\ The Netherlands
}

(Dated: October 25, 2018) 


\begin{abstract}
The predictive simulation of molecular liquids requires models that are not only accurate, but computationally efficient enough to handle the large systems and long time scales required for reliable prediction of macroscopic properties. We present a new approach to the systematic approximation of the first-principles potential energy surface (PES) of molecular liquids using the GAP (Gaussian Approximation Potential) framework. The approach allows us to create potentials at several different levels of accuracy in reproducing the true PES, which allows us to test the level of quantum chemistry that is necessary to accurately predict its macroscopic properties. We test the approach by building potentials for liquid methane $\left(\mathrm{CH}_{4}\right)$, which is difficult to model from first principles because its behavior is dominated by weak dispersion interactions with a significant many-body component. We find that an accurate, consistent prediction of its bulk density across a wide range of temperature and pressure requires not only many-body dispersion, but also quantum nuclear effects to be modeled accurately.
\end{abstract}

\footnotetext{
* max.veit@epfl.ch; Current address: Laboratory of Computational Science and Modeling, Ecole Polytechnique Fédérale de Lausanne, 1015 Lausanne, Switzerland
} 


\section{INTRODUCTION}

The accurate simulation of molecular liquids is a problem of great scientific and industrial importance. We not only would like to be able to test the predictions of our models against experimental benchmarks to see where they need to be refined, but we also need to make predictions for new compounds or mixtures in order to identify the most promising candidates for future applications. When modeling molecular liquids one is typically obliged to trade off accuracy in the description of the potential energy surface and errors due to insufficient sampling. Here we show how one can use function fitting techniques analogous to those in machine learning to create models that reach quantum chemistry accuracy at a much reduced cost. We also break down the total interaction potential into different components that allow us to show explicitly that all components have been modeled sufficiently accurately, and thus we obtain the right answers for the right reasons rather than due to uncontrolled cancellation of errors.

In this work we aim to perform simulations of $a b$ initio quality but with the orders of magnitude boost in computational efficiency afforded by high dimensional regression using machine learning. We create Gaussian approximation potentials (GAPs) [1-3] for liquid methane, the simplest alkane, which is inherently difficult to model because its behavior is dominated by weak dispersion interactions. It is also useful as a stepping stone towards potentials that can model larger hydrocarbons

under more extreme conditions $[4,5]$; such a potential would enable new research in numerous scientific and engineering applications [6-8].

There is a long history of modeling liquids at the atomistic scale with Monte Carlo 
(MC) or molecular dynamics (MD) methods. The interactions between constituent particles are often modeled using analytical potentials, which are a combination of a few simple, physically motivated expressions, such as the venerable Lennard-Jones potential [9] (the 12-6 form, hereafter referred to as L-J) and the many subsequent variations or extensions of this basic form [10-15]. These potentials contain empirical parameters which are usually optimized until the simulations reproduce specific sections of the experimental equation of state.

Recent potentials show a trend of more closely representing the underlying quantum mechanical potential energy surface, for example by adding anharmonic and cross terms to the covalent forces to arrive at a more faithful representation [13, $16,17]$ or even directly fitting the intramolecular [18] or intermolecular $[5,19-22]$ terms to $a b$ initio calculations. However, the analytical parameterized functional forms employed in such potentials remain too simple to represent the underlying potential energy surface faithfully; Figure 1 shows that a pair potential [22] fit directly to coupled-cluster data cannot represent the complex, anisotropic potential energy surface of the methane dimer. It additionally shows a potential that we fit to CCSD(T) using the Gaussian Approximation Potentials (GAP) method [1, 2] (more details in the supporting information) in the full six-dimensional space of mutual dimer orientations (monomers kept rigid); this potential, which we will call the '6-D dimer GAP', is indeed capable of representing the complex anisotropy of the dimer's potential energy surface. Hence, potentials fit with fixed functional forms must represent thermal averages of this surface that are useful for making predictions within a certain range of temperature and pressure; these predictions typically break down once the simulations are taken outside of this range (see Figure 5). It is indicative and somewhat sobering that the most accurate prediction of the density of liquid methane is achieved by the simplest potentials, TraPPE (the coarse united atom version TraPPE-UA [23] and the reduced dimensional version TraPPE-EH [24]). 
Neither attempts to reproduce the actual Born-Oppenheimer potential energy surface; in fact, every effort up to now to capture the potential energy surface by an analytical potential has lead to worse predictions of the liquid density.

Several methods are available that approximate the true quantum potential energy surface. Perhaps the best known of these is density functional theory (DFT) [27], which is generally good at predicting covalent bond energies and intermolecular repulsive interactions. Standard DFT lacks dispersion interactions, however, so these must be added separately [28]. Dispersion correction schemes for DFT are generally inverse-power terms added on to the total DFT energy. They range from terms with fixed semiempirical coefficients [29] to explicitly geometry-dependent terms [30], to terms with coefficients that use information from an existing DFT calculation [31-33]. Many of these schemes, such as DFT-D3 [30] and MBD [33], account for many-body (i.e. beyond pairwise additive) dispersion interactions. This many-body effect has been shown to be crucial for an accurate description of many dispersion-bound systems such as supramolecular complexes [34] and organic crystals [35], though the effects on molecular liquids have not yet been extensively studied - a many-body vdW model (D3 [30]) was included in the water potential of Morawietz et al. [36], but it was not mentioned whether a simple pairwise model would have given different results.

The main drawback of quantum methods that treat electrons explicitly, such as DFT or quantum chemistry, is their computational cost: MD simulations to predict liquid properties routinely require millions of force evaluations on thousands of atoms [7], which would be prohibitive even for today's fastest computers using the most efficient implementations of DFT. Furthermore, MD simulations require force evaluations on many highly correlated configurations. But the Born-Oppenheimer potential energy surface is usually assumed to be smooth and regular, at least in 


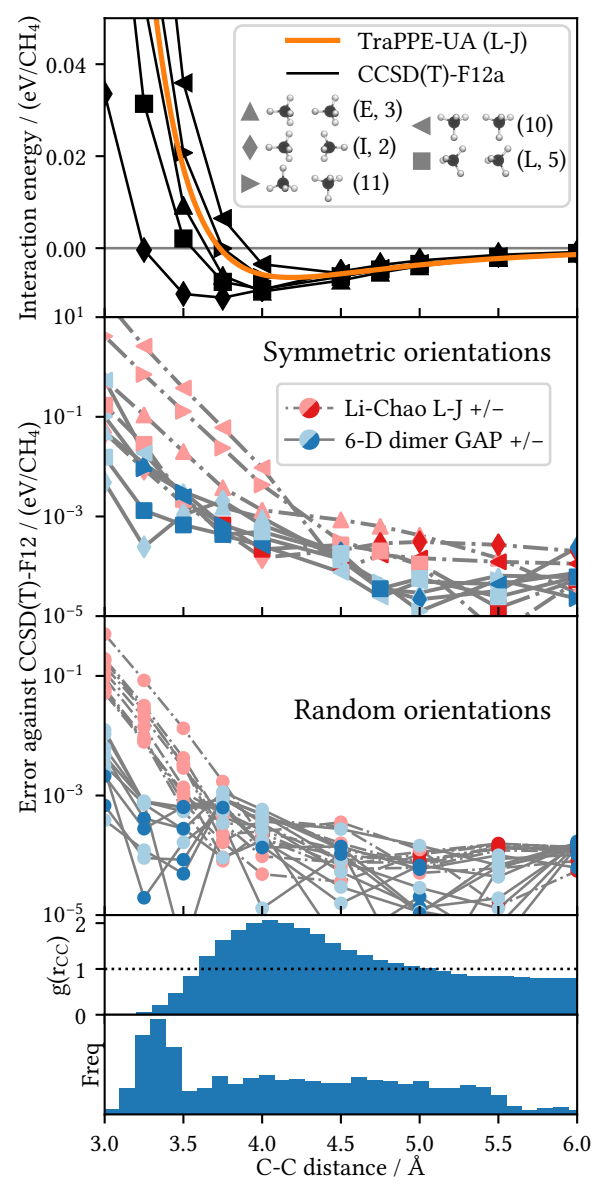

FIG. 1. Top: Interaction energies of the rigid methane dimer in a selection of orientations. The TraPPE united-atom (and therefore isotropic) model [23] is given by the smooth line. Configurations are labeled as in Chao et al. [25] (letters) and Hellmann et al. [21] (numbers). Middle: Errors of two models of the methane dimer energy, the pairwise L-J fit of Li and Chao [22] and a full-dimensional GAP fit, against CCSD(T)-F12 on the same orientations. Bottom: Errors with ten randomly chosen orientations. A pair correlation function at $188 \mathrm{~K}$ and 278 bar and a histogram of the fitting database are given below for reference. Dimer pictures made with VMD [26]. 
the ordinary realm of closed-shell molecules far away from level crossings and other exotic PES irregularities. Thanks to this regularity, highly correlated (similar) configurations will also have highly correlated energies and forces. This correlation can be exploited to greatly reduce the number of force evaluations required for a molecular simulation.

\section{A. Machine learning potentials}

A new generation of potentials aims to exploit this correlation by using machine learning techniques to directly fit the Born-Oppenheimer potential energy surface $[1,37]$. These fits do not constrain the potential's functional form, relying instead on a sufficient sample of existing calculations to be able to regress (fit) these data points in the high-dimensional space of nuclear positions. Such potentials are designed to capture much of the accuracy and flexibility offered by full quantum methods but with a computational efficiency that is many orders of magnitude higher, enabling MD simulations for system sizes and timescales previously only accessible to empirical, analytical potentials.

Machine learning potentials have been applied to a wide variety of systems [38]. In our group, they have been applied to systems ranging from solids such as silicon [1], tungsten [39], iron [40], and boron [41], molecular clusters [3] and liquids [36, 42], and amorphous materials [43]. There is also considerable interest in general, transferable molecular potentials [44] and accurate modeling of liquid water [36]. Recent progress has also been made in modeling across different chemical compounds [45, 46] and even across different classes of materials [47], thus approaching the level of flexibility currently offered by full quantum methods. 


\section{B. Quantum nuclear effects}

Empirical potentials have been fit to reproduce experimental equations of state, so they include quantum nuclear effects implicitly. In contrast, when simulations are done with a systematic approximation of the Born-Oppenheimer potential energy surface, it becomes necessary to account for quantum nuclear effects in an equally systematic manner [48, 49]. These effects are especially important at low temperatures and with light nuclei; their importance in liquid alkanes in particular has long been established [50] and was recently highlighted [51] using quantum mechanically

fitted forcefields. In empirical potentials these effects are typically included in an average way, since they are naturally present in the experimental data used to fit the potentials; some potentials [21] also use a semiempirical or approximate method to include these effects. But in order for a potential to systematically fit the true potential energy surface it cannot include quantum nuclear effects at the level of the fitting, because the true Born-Oppenheimer potential energy surface does not itself include these effects. Thus, fitting methods that include such an average contribution are not fitting the true potential energy surface and are therefore incompatible with the current strategy.

The most common and practical technique for including quantum nuclear effects (ZPVE and nuclear tunneling, but not the nuclear exchange) in MD simulations is via path integral molecular dynamics (PIMD), where the quantum system is represented by $P$ replicas of the classical system, corresponding atoms being joined across the replicas by harmonic springs in a ring-polymer structure [48, 52-54]. Recent techniques, including improved stochastic thermostats [55-57] and ring polymer contraction [58], are making PIMD practical even for large systems and more expensive potentials such as the ones employed in this work.

Despite these new developments, ab initio liquid simulation remains a challenge. 
The process of designing a machine learning potential for a new material, especially for amorphous or liquid simulation, is still a laborious manual process. In this work we develop a methodology that will eventually serve as a foundation for more systematic, perhaps even automated, development of potentials for more complex molecular liquids.

\section{MODEL DEVELOPMENT METHODOLOGY}

Fundamental to this methodology is a strategy common to most successful potentials for molecular systems: The energy of the system is decomposed into several terms that each represents a different physical interaction. From the point of view of a physics-based analytical potential, this decomposition is useful because the different physical interactions will typically have different functional forms, and it makes sense to parameterize them separately. From the point of view of a machine learning potential, the main advantage of an energy decomposition scheme is that it separates physical effects that take place at different length and energy scales and prevents the larger effects from overwhelming the smaller ones; while the smaller components might not be important in reproducing the total energy, other important observables (such as the density or the diffusivity) might well weight these contributions much higher. By controlling the accuracy of the several components separately it is possible to achieve good accuracy on any property of interest.

In a molecular liquid such as methane, the primary separation in energy scales is between the strong intramolecular (covalent) interactions and the weak intermolecular (noncovalent) interactions. These two types of interactions are easy to separate and have characteristic energy scales that are orders of magnitude apart. The second separation we will employ here is motivated by the length scales of the interactions, as machine learning potentials tend to work best for fitting functions that vary on 
a single length scale. In methane, the dispersion (van der Waals) interaction is very long-ranged, being still relevant at C-C distances as large as $15 \AA$, but the various repulsive interactions generated by electron cloud overlap die out by $\mathrm{C}-\mathrm{C}$ distances of $5 \AA$. The long-range electrostatic energy is predicted (by most empirical potentials) to be negligible for pure methane, which is neutral and nonpolar, so it is not explicitly included. The energy equation we will use is therefore:

$$
E_{\text {total }}=E_{1 \mathrm{~b}}+E_{\text {repulsion, }, \mathrm{b} 1 \mathrm{~b}}+E_{\text {dispersion }}
$$

where the ' $1 b$ ' (one-body) energy is the covalent part and 'b1b' signifies the intermolecular (beyond one-body) part. The second term here is computed from DFT beyond-one-body interactions, while the dispersion term is computed separately.

Besides making it easier to fit the potential, another advantage of this approach is that it allows us to capture some of the underlying physics of the system. Some recent analytical potentials take the approach of more closely representing the underlying physics by extracting forcefield parameters from fundamental physical quantities such as the electron density. Models using this approach include the Slater-ISA model of Van Vleet et al. [59] and the biomolecular force field of Cole et al. [60]. The IPML model of Bereau et. al. [61] goes one step further by using machine learning to efficiently predict these properties across chemical compound space. While the physical interpretability of these models is appealing, it comes at the cost of sacrificing a bestpossible fit to the true quantum potential energy surface. In the present potential, by capturing the simple, physically motivated parts of the energy expression by simple analytical forms and fitting the complex, nonanalytical parts as corrections on top of these, we use physics to guide our description of the interaction while maintaining complete flexibility of the functional form. 

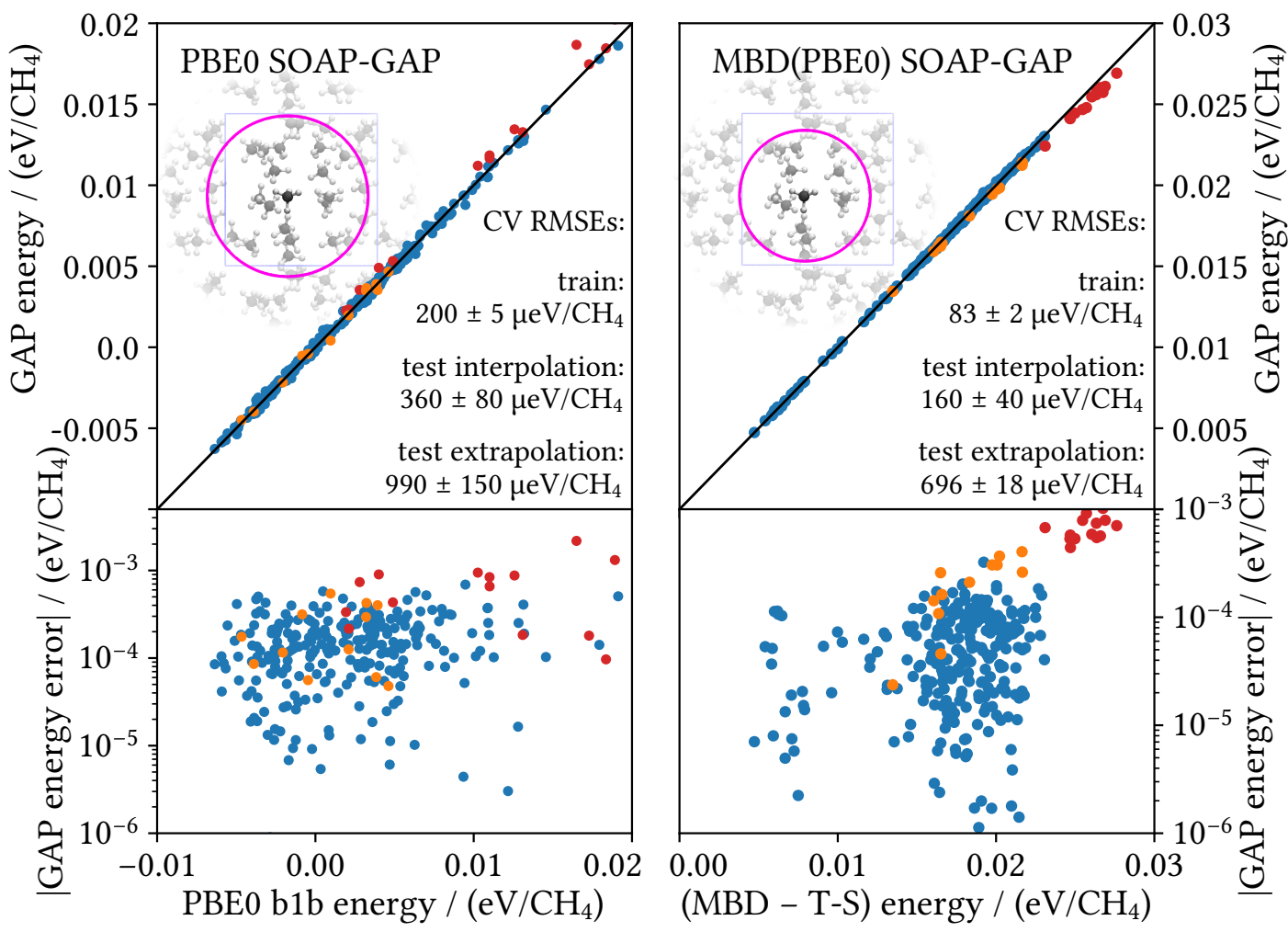

FIG. 2. The PBE0 and MBD(PBE0) SOAP-GAP fits on 258 cell interaction (beyond onebody, 'b1b') energies and (only for PBE0) corresponding forces. Top: Correlation plots with the line $y=x$ of perfect correlation. Bottom: Errors on a logarithmic scale. The blue points represent the training set. The orange points represent the interpolation test set and the red points represent the extrapolation test set (color online), neither of which was used in training the model.

\section{A. Machine learning model}

To fit the nonanalytical components of the potential for bulk methane - the second part of Equation (1) - we use the GAP method [1, 2] with the SOAP kernel [62], both developed and used by our group to fit complex, many-body potentials. The SOAP- 
GAP potentials were fit to DFT $[63,64]$ b1b (beyond-one-body interactions; the monomers were computed separately and subtracted from the total cell) energies and forces, computed on samples of bulk methane taken from MD trajectories under liquid conditions run using a classical potential (OPLS/AMBER $[65,66]$ ) at a temperature of $188 \mathrm{~K}$ and five pressures ranging from 0 bar to $400 \mathrm{bar}$, thus covering the entire range of pressures encountered in the subsequent GAP MD simulations. The resulting training set consisted of a wide range of densities; see Figure 3. However, the typical densities encountered during a simulation at $110 \mathrm{~K}$ in the same pressure range fall partly outside this range, exercising both the model's interpolation and extrapolation capabilities. To validate these capabilities, independent samples were drawn from OPLS/AMBER simulations at both temperatures, with several samples taken from each of the state points where classical results are shown in Figure 5 below. The histogram of the densities of these test sets is also shown in Figure 3. Based on the position of these distributions relative to the test set, the 12 test samples taken at $188 \mathrm{~K}$ were labeled the 'interpolation' test set and the 14 samples from $110 \mathrm{~K}$ were labeled the 'extrapolation' test set.

The DFT calculations on all cells were done using CASTEP [67]. Two functionals were used, PBE [68] and the hybrid GGA functional PBE0 [69]. The GAP fits were done using the SOAP descriptor [62], resulting in two models called 'PBE SOAP-GAP' and 'PBE0 SOAP-GAP'. The performance of the PBE0 SOAP-GAP is assessed in Figure 2, which indicates good reproduction of both energies and forces on the training set. Since GAP is a statistical learning method, this is usually a good measure of how the method will perform on similar geometries. The interpolation performance indicates some degree of overfitting, though, while the extrapolation performance is significantly worse - but the model still achieves an error of less than $1 \mathrm{meV}$ per molecule on systems that were never included in the fit. The variability of 


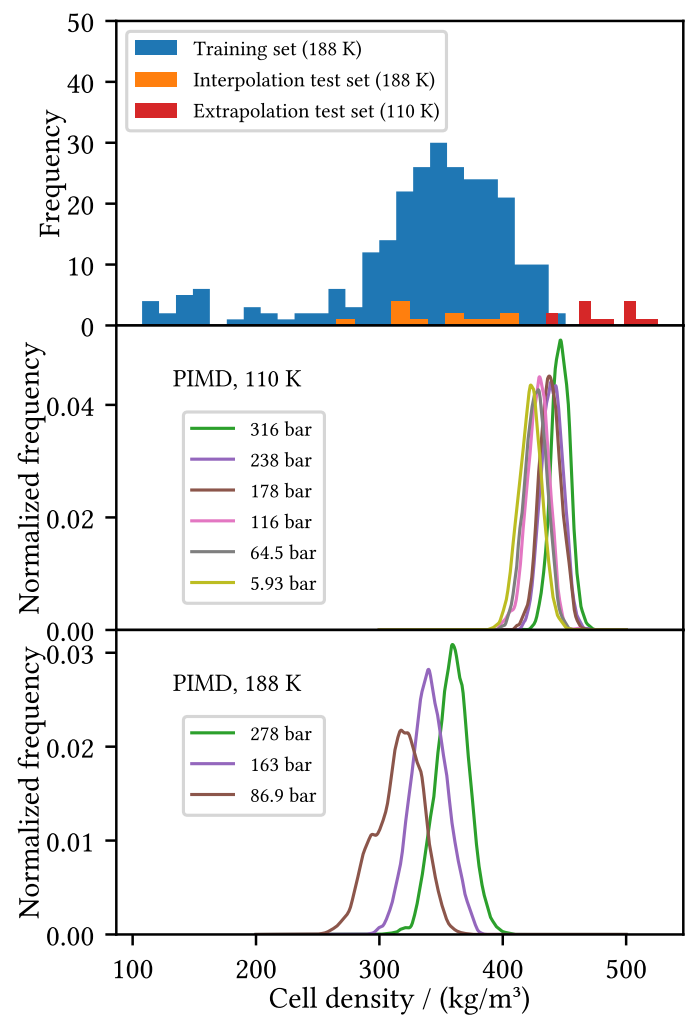

FIG. 3. Histograms over mass density of the cells in the training and two test sets, interpolation and extrapolation. The distributions of densities encountered in the subsequent PIMD simulations with the (PBE0 SOAP)/COMPASS + T-S + MBD(PBE0) SOAP model (see below) are shown below for comparison.

this error measure was assessed with a cross-validation (CV) procedure: Ten disjoint sets of twelve points each were selected from the training data, and each in turn substituted with the interpolation test set to train ten additional GAP models. The numbers reported in Figure 2 are obtained as the mean and standard deviation of the errors across this set of eleven GAPs, with the withheld points standing in for the interpolation test set in each validation GAP. The errors on the forces show the same 
pattern: The training set error is $(6.56 \pm 0.03) \mathrm{meV} / \AA$, the interpolation test set er-

ror is $(6.8 \pm 0.6) \mathrm{meV} / \AA$, and the extrapolation test set error is $(8.71 \pm 0.05) \mathrm{meV} / \AA$. Plots of the forces for the similar PBE SOAP-GAP, along with its energy and force errors, can be found in the supporting information.

\section{B. Dispersion model}

The dispersion component, the third term in Equation (1), was accounted for using two levels of theory. The first was the pairwise correction of Tkatchenko and Scheffler [32]. This method uses relative atomic volumes from a Hirshfeld partitioning [70] of the electron density, an idea introduced by Becke and Johnson [31], and relates them to free-atom dispersion coefficients (those computed by Chu and Dalgarno [71] were used here). Recomputing the Hirshfeld volumes for each step of an MD simulation would be impractically expensive, as that would require a new DFT calculation at each step. Instead, the first level of theory only uses the per-element average of the relative Hirshfeld volumes across the sample of DFT cells. The dispersion correction can then be applied as an analytical pair potential whose form and parameters are fixed throughout the simulation, a scheme hereafter termed simply 'T-S'.

The second level of theory is the MBD, or many-body dispersion, method [33, 72]. Despite the greater complexity of the MBD approach, it can still be viewed as a correction on top of the pairwise Tkatchenko-Scheffler interaction. Thus, another SOAP-GAP was fit to the difference between the MBD energies only and the (fixed) T-S term as the baseline, once each for PBE and PBE0 Hirshfeld volumes. This model, termed 'MBD(PBE) SOAP-GAP' (and the corresponding 'MBD(PBE0) SOAP-GAP'), accounts for relatively short-ranged many-body effects. The dispersion 
energy term from Equation (1) therefore becomes:

$$
E_{\text {dispersion }}=E_{\mathrm{T}-\mathrm{S}}+E_{\mathrm{MBD} \text { SOAP-GAP. }}
$$

The MBD SOAP-GAP also implicitly accounts for the variability of the Hirshfeld volumes that was neglected in the fixed T-S model: The SOAP descriptor is sensitive to the intramolecular and short-range geometrical factors that (presumably) also account for the variability of these volumes. The MBD(PBE0) fit is likewise assessed in Figure 2, showing that both its interpolation and extrapolation performance is similar to that of the PBE0 SOAP-GAP.

Finally, a complete model for liquid methane must also include an intramolecular component (the first term in Equation (1)). Two empirical potentials are considered for this purpose: AMBER [66] includes only harmonic bond and angle terms, while COMPASS [13] includes higher-order anharmonic and cross-coupling terms. Both models were tested in order to help measure the influence of such effects (anharmonic and cross-coupling) on the predicted properties, especially with the inclusion of quantum nuclear effects.

\section{RESULTS}

The first test of the accuracy and applicability of any potential for liquids is how well it reproduces the experimental equation of state. While most empirical potentials (for example OPLS [65]) are fit to reproduce experimental thermodynamic data, the fitting conditions are often only a single state point per material, usually standard temperature and pressure. Some potentials, like TraPPE [23], are fit to reproduce thermodynamic data across a wide range of state points, in this case by fitting coexistence curves. Therefore, a wide range of temperature and pressure conditions were chosen to test the accuracy of the potentials considered. Two isotherms were 
chosen where experimental data was available (from Goodwin and Prydz [73]): At $110 \mathrm{~K}$, density measurements were available at 5.93 bar, 64.5 bar, 116 bar, 179 bar, 238 bar and 316 bar[74]. At $188 \mathrm{~K}$, density measurements were available at 86.9 bar, 163 bar and 278 bar[74].

Four GAPs were chosen for testing: The 'PBE SOAP-GAP' model with both fixed T-S ('+ T-S') and MBD ('+ T-S + MBD(PBE) SOAP') dispersion, the 'PBE0 SOAP-GAP $+\mathrm{T}-\mathrm{S}+\mathrm{MBD}(\mathrm{PBE} 0)$ SOAP-GAP', and the 6-D dimer GAP described above and shown in Figure 1. The dimer GAP and all of the SOAP-GAP models were first tested at the state point $110 \mathrm{~K}$ and 316 bar using a "smart sampling" colored-noise thermostat for efficient equilibration [75]. The convergence of the results towards the experimental density is illustrated in Figure 4; for brevity, all the 'SOAP-GAP' models are labeled simply with 'SOAP'. A selection of empirical potentials is also shown: TraPPE-UA [23], TraPPE-EH [24], OPLS/AMBER [65, 66] (a combination of OPLS-AA with the AMBER intramolecular parameters), and the Li-Chao L-J fit [22].

The density predictions are shown against the error of the underlying quantum model computed on a sample of dimers (the same one used to fit the 6-D dimer GAP), with CCSD(T)-F12 taken as the reference. In the case of the GAPs, the statistical uncertainty introduced by the fits is shown and added to the systematic uncertainty already given by the quantum model. In the case of the empirical model, the error is taken to be entirely systematic.

Evidently, the predictions for the density at both state points improve as the dispersion model is made more accurate as measured on the methane dimer, an improvement that is reflected in the dimer error measure. Adding the MBD SOAPGAP lowers the density by $15 \mathrm{~kg} / \mathrm{m}^{3}$, improving the prediction by $3.4 \%$ with respect to experiment; the short-range improvement offered by switching to PBE0 gives a 


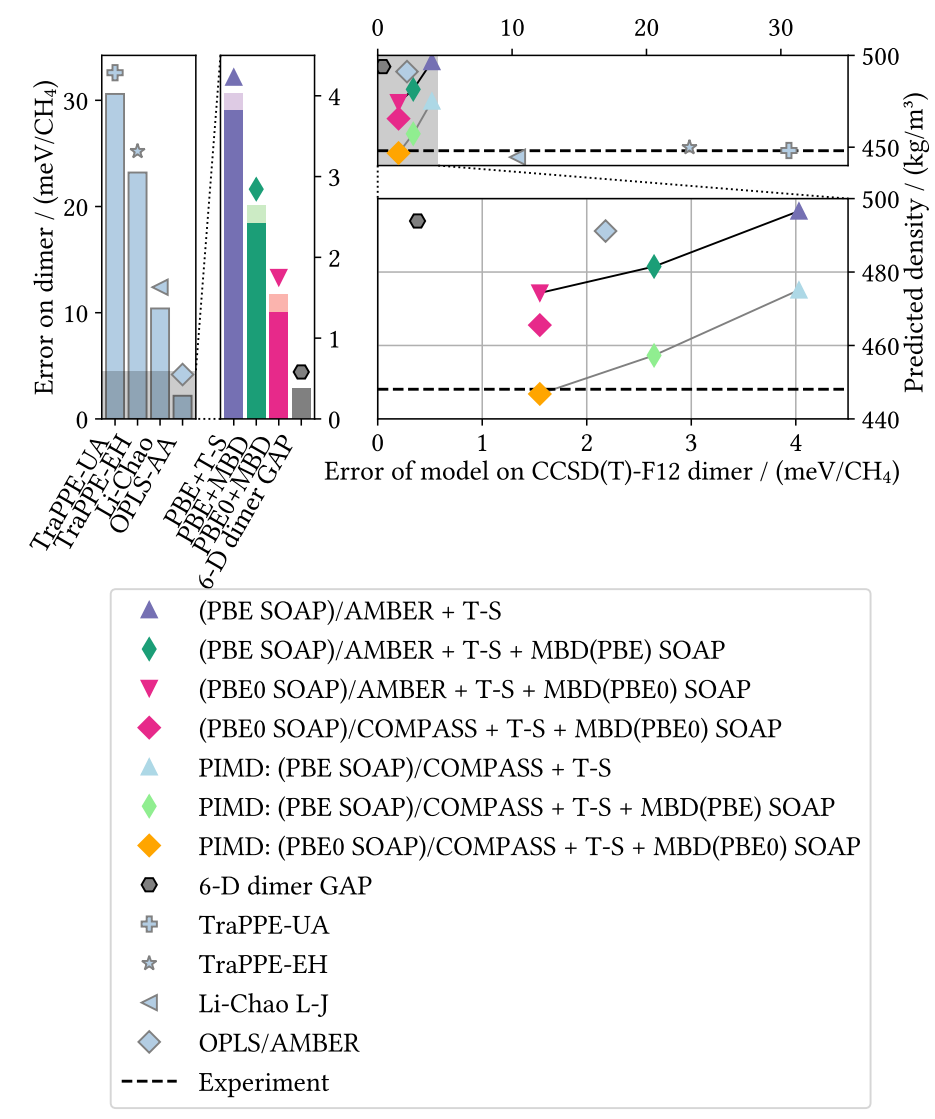

FIG. 4. Systematic convergence of the density predictions of the bulk SOAP-GAPs as the dispersion model is improved; single state point at $110 \mathrm{~K}$ and 316 bar: Convergence of the classical simulations is shown by the black connecting line, PIMD simulations with the grey line. The uncertainties on the density are smaller than the sizes of the symbols. The "dimer error" figure is computed against $\operatorname{CCSD}(\mathrm{T})-\mathrm{F} 12$ on the sample of dimers used to train the 6-D dimer GAP. In the right-hand bar plot, solid bars represent the systematic errors due to the underlying quantum model and the pastel bars on top represent the statistical errors introduced by the GAP fit. In the left-hand bar plot, the bars represent the (systematic) error of the analytical model against the same coupled-cluster reference. 
further $7.2 \mathrm{~kg} / \mathrm{m}^{3}(1.6 \%)$ improvement. Given the large influence many-body effects appear to have on the density, it is perhaps not surprising that the general dimer GAP gives such a bad prediction, $45.8 \mathrm{~kg} / \mathrm{m}^{3}$ or $10.2 \%$ higher than experiment. While the figure indicates that there are still effects not included by the dimer measure of accuracy - especially the intramolecular potential and many-body (beyond dimer) effects - it still shows a general trend of improvement of the potential's predictions as it more accurately represents the underlying potential energy surface. Crucially, this is a trait not shared by empirical potentials - TraPPE, OPLS/AMBER and the Li-Chao L-J - which show the opposite behavior. The models with the best density prediction are also the ones with the worst performance on the methane dimer, indicating that they must achieve their accuracy by a large cancellation of errors. (It should also be noted that the accuracy of the Li-Chao L-J model is without explicit quantum nuclear effects, even though the potential was ostensibly fitted to the Born-Oppenheimer potential energy surface of the dimers, thus further muddling the picture for empirical potentials).

The quantum nuclear effect was assessed in an explicit way, using a PIMD simulation using the PIGLET thermostat $[56,57]$. With this effect included, the best model ('PBE0 SOAP + T-S + MBD(PBE0) SOAP') delivers a prediction within $0.3 \%$ (nearly within simulation uncertainty) of the experimental number. This decrease in density is of the same order of magnitude as that reported in Pereyaslavets et al. [51], though with this potential the effect is smaller $-4.2 \%$ instead of $9 \%$. Figure 5 shows that the size of the effect is roughly the same across the $110 \mathrm{~K}$ isotherm, so even at the $112 \mathrm{~K}, 1$ bar state point used in that study we would expect to see a somewhat smaller effect. The decrease also intuitively contrasts with the finding that, in the gas phase of methane, zero-point vibrational contributions increase the molecular $C^{6}$ (first pairwise dispersion) coefficient and hence the strength of the intermolecular attraction [21, 76, 77]. Evidently, this effect is overpowered in the condensed phase 
by an increase in repulsion leading to a decrease in the density; a likely candidate is the increase in molecular size due to the same zero-point vibrational energy lengthening the bonds [50]. The exact mechanism is still unclear, though, and the ab initio quality potentials presented here provide the means necessary for further study of this effect.

The performance of the models across both of the experimental isotherms is shown in Figure 5. For comparison, a selection of analytical potentials was tested at all the state points at $110 \mathrm{~K}$ and $188 \mathrm{~K}$ with experimental data, plus an additional point at 400 bar for each isotherm to show the high-pressure trend. In addition to the potentials shown in Figure 4, the figure also shows the L-J baseline and COMPASS [13]. Note in particular that the empirical all-atom potentials all shift with respect to experiment between the two isotherms. Most models, the GAPs included, have more trouble reproducing the density at the $188 \mathrm{~K}$ isotherm, perhaps because of the proximity of the lowest-pressure point to the critical point (190.58 K and 46.04 bar [78]). Only the united-atom model TraPPE-UA maintains accuracy across the whole space of conditions covered, with the explicit-hydrogen description TraPPE-EH closely following in consistency. The series of SOAP-GAP potentials delivers predictions of increasing accuracy, in correlation with the accuracy on the dimer. Despite the relatively large statistical fluctuations in the PIMD SOAP-GAP density predictions, the model is still more consistently accurate (comparing across both isotherms) than any other model fit to the quantum PES, especially with the explicit inclusion of quantum nuclear effects. It thus appears essential to include quantum nuclear effects in order to make accurate predictions with a potential fitted to the Born-Oppenheimer quantum potential energy surface. Other potentials that achieve agreement with experiment without explicit treatment of these effects must be incorporating them into the potential energy surface itself, which is at odds with our stated goal of achieving 

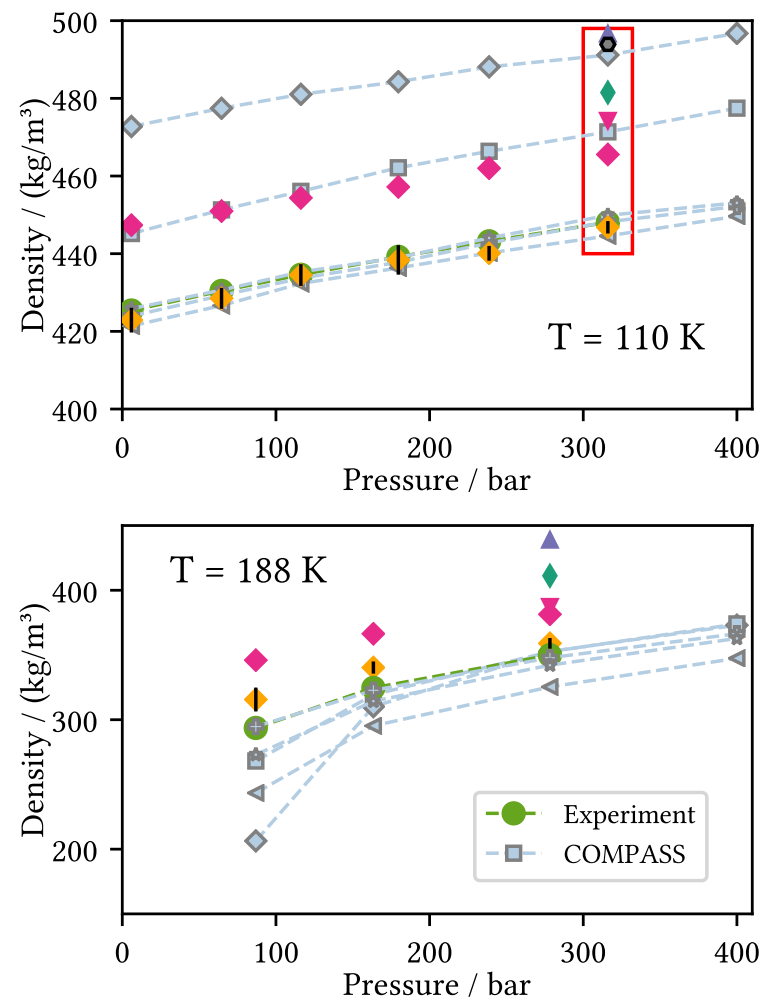

FIG. 5. Equation of state at two temperatures, $110 \mathrm{~K}$ and $188 \mathrm{~K}$, as predicted by various atomistic models. The bulk SOAP-GAPs with different dispersion models are shown, as is the 6-D dimer GAP. All-atom empirical models are shown in gray. Experimental data from Goodwin and Prydz [73]. The small black lines are error bars on the PIMD simulations computed using the blocking method described in the supporting information. Refer to the legend of Figure 4 for symbols previously defined.

the agreement with experiment in an ab initio manner by best fitting the potential energy surface.

In summary, while TraPPE potentials obtain their accuracy by fitting to experimental data across wide ranges of temperature and pressure, the SOAP-GAP 
potentials obtain their accuracy by fitting to the underlying quantum mechanical description of matter and systematically converge to within $0.5 \%$ of the experimental value as their description is improved (while TraPPE-EH, while being in some sense a more accurate model than TraPPE-UA by describing the anisotropy of the methane molecule, delivers worse predictions especially at the $188 \mathrm{~K}$ isotherm). Additionally, even the current best SOAP-GAP model still has several routes of potential improvement that would not be open to a fixed-form analytical potential, such as changing the intramolecular model for a more accurate, fitted one or improving the dimer description to the coupled-cluster dimer GAP level (which can be done using existing techniques, e.g. by adding a further two-body correction to the SOAP-GAP model $[3,42])$.

\section{DISCUSSION}

The fitting and testing of the SOAP-GAP and dimer potentials for liquid methane reveal three key findings for the description of molecular liquids: First, many-body effects - not only within the dimer, but also beyond-dimer effects - are essential, especially in the short range, for obtaining an accurate description of the bulk density. Second, an explicit description of quantum nuclear effects is equally important, especially at the temperatures and pressures considered here. Third, systematic measures of the accuracy of the potential (such as the dimer error measure presented here) are a good guide to improving systematically fitted potentials toward convergence with the experimental results, a goal which the (PBE0 SOAP-GAP) + T-S $+\mathrm{MBD}(\mathrm{PBE} 0)$ SOAP-GAP model presented here comes close to achieving.

The methodology presented here is a new, physics-based, systematic path toward creating exceptionally accurate potentials for molecular liquids. The methodology is expected to generalize in a straightforward way to longer hydrocarbons, where 
the more difficult part then becomes the description of intramolecular interactions. Furthermore, the ideas presented here could be extended to other types of longrange interactions, such as electrostatics and induction, in order to extend accurate machine learning potentials to a wider variety of molecular liquids.

\section{COMPUTATIONAL METHODS}

\section{A. Gaussian processes}

The GAP machine learning method used to fit the potential energy is based on Gaussian process regression and is part of the family of kernel learning methods $[1,2]$. Such methods perform linear fits in a transformed data space: The nonlinearity of the function is now captured in a kernel function, also called a similarity or covariance function, which usually measures the similarity between two local atomic environments (although they can also be designed to capture long-range and global properties).

Formally, the potential energy suface is represented as a Gaussian process [79, 80]. The covariance matrix of this process is formulated to use the information provided by quantum calculations, i.e. total energies and derivatives, in a natural way through linear operations on the kernel. This allows the Gaussian process to provide a smooth approximation of the potential energy surface, as sampled by the quantum data points, in the high-dimensional space of atomic or molecular environments using just a linear combination of kernels; for example, the local energy of an atom $i$ is given by:

$$
\varepsilon_{i}=\sum_{j} \alpha_{j} k\left(\mathbf{d}_{j}, \mathbf{d}_{i}\right)
$$

where the $\mathbf{d}$ are descriptors of local atomic environments, $k$ designates the covariance or kernel function, and the weights $\alpha$ are determined by a regularized least-squares 
linear fit to the quantum mechanical training data (in this view, the predictions of Gaussian processes are the same as those given by kernel ridge regression (KRR) with a radial basis) [2]. In GAP, the sum runs over a subset of representative configurations in the training set, allowing the fitting to scale linearly with the number of input data points.

The most successful kernel function for condensed-phase GAP has been the SOAP kernel [62], which takes the similarity between local atomic environments. The environment of atom $i$ is represented by a neighbor density $\rho_{i}(\mathbf{r})$, defined as a sum of Gaussians placed on each neighboring atom, multiplied by a spherical cutoff function which smoothly takes the density to zero outside some cutoff radius. The kernel between two environments is defined as the integral over all possible mutual rotations of the square of the overlap between the two neighbor densities, thus making the kernel obey the same symmetries as the local energy: Invariance to translations (environments are atom-centred), permutations (from summing like atoms in the neighbor density), and rotations (from the rotational integration).

In practice, the integration over rotations can be done analytically by expanding each neighbor density in spherical harmonics and radial basis functions:

$$
\rho_{i}(\mathbf{r})=\sum_{n l m} c_{n l m}^{(i)} g_{n}(r) Y_{l m}(\hat{\mathbf{r}}),
$$

computing the power spectrum elements

$$
p_{n n^{\prime} l}^{(i)}=\frac{1}{\sqrt{2 l+1}} \sum_{m} c_{n l m}^{(i)}\left(c_{n^{\prime} l m}^{(i)}\right)^{\dagger},
$$

and summing in order to obtain the covariance function:

$$
k_{0}\left(\rho_{i}, \rho_{j}\right)=\sum_{n n^{\prime} l} p_{n n^{\prime} l}^{(i)} p_{n n^{\prime} l}^{(j)}
$$


which is then normalized to obtain a proper kernel and optionally raised to some power $\zeta>1$ to increase the sensitivity to changes in the local environment $[2,62]$.

Note here that the local environment is effectively represented by a set of numbers $\mathbf{p}^{(i)}$, which can be interpreted as a "descriptor" or even "feature vector" of the environment. Many other kernels are formulated in terms of such descriptors, such as the 6-D dimer kernel described in the supporting information.

The GAP models used in this study were all fit and evaluated using the libAtoms/QUIP package [81]. The GAP code can be downloaded at http://www . libatoms.org/gap/gap_download.html, with a precompiled version available through Docker at https://hub.docker.com/r/libatomsquip/quip/.

\section{B. MD simulations}

The MD simulations were run using QUIP [81] and i-PI [82] via LAMMPS [83, 84]. The former used the adaptive Langevin thermostat of Jones and Leimkuhler [85] and a Hoover-Langevin barostat [86] while the latter used a thermostat based on the generalized Langevin equation (GLE, otherwise known as colored-noise thermostats), namely the "smart sampling" method of Ceriotti, Bussi, and Parrinello [75], for the classical simulations and PIGLET $[56,57]$ for the PIMD simulations. The initial configurations for all simulations were generated using Packmol [87].

The analytical potentials were run in LAMMPS [83] with a Langevin thermostat [88] and a Nosé-Hoover barostat [89-93] with the MTK correction [94]. For potentials with a Coulomb component (OPLS/AMBER and COMPASS), the contributions beyond the cutoff were calculated with the particle-particle particle-mesh (PPPM) method [95]. 


\section{Dimer fits}

The coupled-cluster $\operatorname{CCSD}(\mathrm{T})$ energies of the methane dimer were computed in a similar way as described in Gillan et. al. [3] (explained in more detail in the supporting information), up to the level of $\operatorname{CCSD}(\mathrm{T})-\mathrm{F} 12$ [96-98]. The energies were corrected for basis-set superposition error (BSSE) using the Boys-Bernardi counterpoise procedure [99]. Calculations were done using the MOLPRO suite of programs [100-103]. The Atomic Simulation Enviroment (ASE) [104] was used to generate and manipulate geometries. For the dimer error numbers used in Figure 4, energies (PBE and PBE0) were computed with PSI4 [105] and the Hirshfeld partitioning [70] was done using HORTON [106-109].

The geometries for the randomly chosen orientations were directly sampled from a liquid MD simulation (details in the supplementary information). Ten orientations were sampled and each used to produce a binding curve with regularly spaced dimer separations.

Finally, all the graphics in this paper were made using Matplotlib [110]; the analysis was done within the Jupyter interactive computing enviroment with the IPython kernel [111].

\section{ACKNOWLEDGMENTS}

M.V. acknowledges Shell Global Solutions International B.V. for funding, as well as support from the EPSRC Centre for Doctoral Training in Computational Methods for Materials Science (under grant number EP/L015552/1). This work used the ARCHER UK National Supercomputing Service (http://www.archer.ac.uk) under the UCKP Consortium, EPSRC grant number EP/P022596/1. We gratefully acknowledge the assistance of Venkat Kapil in preparing the GLE and PIMD simu- 
lations.

\section{SUPPORTING INFORMATION}

Dimer fit details, parameters for the DFT and quantum chemistry calculations, GAP fitting command lines, and MD simulation trajectories and parameters are given in the supporting information.

The GAP definition files and parameter files required to reproduce the MD simulations in this work are available online at https://doi.org/10.17863/CAM.26364.

[1] A. P. Bartók, M. C. Payne, R. Kondor, and G. Csányi, Phys. Rev. Lett. 104, 136403 (2010).

[2] A. P. Bartók and G. Csányi, Int. J. Quantum Chem. 115, 1051 (2015).

[3] M. J. Gillan, D. Alfé, A. P. Bartók, and G. Csányi, J. Chem. Phys. 139, 244504 (2013), arXiv:1303.0751v1.

[4] G. Kaminski, E. M. Duffy, T. Matsui, and W. L. Jorgensen, J. Phys. Chem. 98, 13077 (1994).

[5] J. M. Hayes, J. C. Greer, and D. A. Morton-Blake, J. Comput. Chem. 25, 1953 (2004).

[6] L. Spanu, D. Donadio, D. Hohl, E. Schwegler, and G. Galli, Proc. Natl. Acad. Sci. 108, $6843(2011)$.

[7] R. S. Payal, S. Balasubramanian, I. Rudra, K. Tandon, I. Mahlke, D. Doyle, and R. Cracknell, Mol. Simul. 38, 1234 (2012).

[8] F. Hansen, K. Herwig, B. Matthies, and H. Taub, Phys Rev Lett 83, 2362 (1999). 
[9] J. E. Lennard-Jones, Proc. Phys. Soc. 43, 461 (1931).

[10] R. A. Buckingham and J. Corner, Proc. R. Soc. A Math. Phys. Eng. Sci. 189, 118 (1947).

[11] S. J. Weiner, P. A. Kollman, D. T. Nguyen, and D. A. Case, J. Comput. Chem. 7, 230 (1986).

[12] W. L. Jorgensen, J. D. Madura, and C. J. Swenson, J. Am. Chem. Soc. 106, 6638 (1984).

[13] H. Sun, J. Phys. Chem. B 102, 7338 (1998).

[14] S. J. Stuart, A. B. Tutein, and J. A. Harrison, J. Chem. Phys. 112, 6472 (2000).

[15] M. P. Allen and D. J. Tildesley, Computer simulation of liquids (Clarendon Press, Oxford, 1989).

[16] J. R. Maple, M.-J. Hwang, T. P. Stockfisch, U. Dinur, M. Waldman, C. S. Ewig, and A. T. Hagler, J. Comput. Chem. 15, 162 (1994).

[17] N. L. Allinger, K. Chen, and J.-H. Lii, J. Comput. Chem. 17, 642 (1996).

[18] A. E. A. Allen, M. C. Payne, and D. J. Cole, J. Chem. Theory Comput. 14, 274 (2018).

[19] D. H. Gay, H. Dai, and D. R. Beck, J. Chem. Phys. 95, 9106 (1991).

[20] J.-P. Jalkanen, T. A. Pakkanen, Y. Yang, and R. L. Rowley, J. Chem. Phys. 118, $5474(2003)$.

[21] R. Hellmann, E. Bich, and E. Vogel, J. Chem. Phys. 128, 214303 (2008).

[22] A. H.-T. Li and S. D. Chao, J. Chinese Chem. Soc. 63, 282 (2016).

[23] M. G. Martin and J. I. Siepmann, J. Phys. Chem. B 102, 2569 (1998).

[24] B. Chen and J. I. Siepmann, J. Phys. Chem. B 103, 5370 (1999).

[25] S.-W. Chao, A. H.-T. Li, and S. D. Chao, J. Comput. Chem. 30, 1839 (2009).

[26] W. Humphrey, A. Dalke, and K. Schulten, J. Mol. Graph. 14, 33 (1996).

[27] R. M. Martin, Electronic structure : basic theory and practical methods, 1st ed. (Cam- 
bridge University Press, Cambridge, UK, 2008).

[28] S. Grimme, A. Hansen, J. G. Brandenburg, and C. Bannwarth, Chem. Rev. 116, 5105 (2016).

[29] S. Grimme, J. Comput. Chem. 27, 1787 (2006).

[30] S. Grimme, J. Antony, S. Ehrlich, and H. Krieg, J. Chem. Phys. 132, 154104 (2010).

[31] A. D. Becke and E. R. Johnson, J. Chem. Phys. 124, 14104 (2006).

[32] A. Tkatchenko and M. Scheffler, Phys. Rev. Lett. 102, 073005 (2009).

[33] A. Tkatchenko, R. A. DiStasio, R. Car, and M. Scheffler, Phys. Rev. Lett. 108, 236402 (2012).

[34] M. A. Blood-Forsythe, T. Markovich, R. A. DiStasio, R. Car, and A. Aspuru-Guzik, Chem. Sci. 7, 1712 (2016), arXiv:1509.00480.

[35] A. G. Shtukenberg, Q. Zhu, D. J. Carter, L. Vogt, J. Hoja, E. Schneider, H. Song, B. Pokroy, I. Polishchuk, A. Tkatchenko, A. R. Oganov, A. L. Rohl, M. E. Tuckerman, and B. Kahr, Chem. Sci. 8, 4926 (2017).

[36] T. Morawietz, A. Singraber, C. Dellago, and J. Behler, Proc. Natl. Acad. Sci. 113, 8368 (2016), arXiv:1606.07775.

[37] J. Behler and M. Parrinello, Phys. Rev. Lett. 98, 146401 (2007).

[38] M. Rupp, O. A. von Lilienfeld, and K. Burke, J. Chem. Phys. 148, 241401 (2018).

[39] W. J. Szlachta, A. P. Bartók, and G. Csányi, Phys. Rev. B 90, 104108 (2014).

[40] D. Dragoni, T. D. Daff, G. Csányi, and N. Marzari, Phys. Rev. Mater. 2, 13808 (2018).

[41] V. L. Deringer, C. J. Pickard, and G. Csányi, Phys. Rev. Lett. 120, 156001 (2018).

[42] A. P. Bartók, M. J. Gillan, F. R. Manby, and G. Csányi, Phys. Rev. B - Condens. Matter Mater. Phys. 88, 054104 (2013).

[43] V. L. Deringer and G. Csányi, Phys. Rev. B 95, 094203 (2017).

[44] T. Bereau, D. Andrienko, and O. A. Von Lilienfeld, J. Chem. Theory Comput. 11, 
3225 (2015), arXiv:1503.05453v2.

[45] S. De, A. P. Bartók, G. Csányi, and M. Ceriotti, Phys. Chem. Chem. Phys. 18, 13754 (2015), arXiv:1601.04077.

[46] G. Montavon, M. Rupp, V. Gobre, A. Vazquez-Mayagoitia, K. Hansen, A. Tkatchenko, K.-R. Müller, and O. Anatole von Lilienfeld, New J. Phys. 15, 095003 (2013).

[47] A. P. Bartók, S. De, C. Poelking, N. Bernstein, J. R. Kermode, G. Csányi, and M. Ceriotti, Sci. Adv. 3, e1701816 (2017), arXiv:1706.00179.

[48] T. E. Markland and M. Ceriotti, Nat. Rev. Chem. 2, 0109 (2018), arXiv:1803.01037.

[49] V. Kapil, J. Behler, and M. Ceriotti, J. Chem. Phys. 145, 234103 (2016).

[50] E. Balog, A. L. Hughes, and G. J. Martyna, J. Chem. Phys. 112, 870 (2000).

[51] L. Pereyaslavets, I. Kurnikov, G. Kamath, O. Butin, A. Illarionov, I. Leontyev, M. Olevanov, M. Levitt, R. D. Kornberg, and B. Fain, Proc. Natl. Acad. Sci. 115, 8878 (2018).

[52] D. Chandler and P. G. Wolynes, J. Chem. Phys. 74, 4078 (1981).

[53] S. Habershon, D. E. Manolopoulos, T. E. Markland, and T. F. Miller, Annu. Rev. Phys. Chem. 64, 387 (2013).

[54] S. Biermann, D. Hohl, and D. Marx, Solid State Commun. 108, 337 (1998).

[55] M. Ceriotti, M. Parrinello, T. E. Markland, and D. E. Manolopoulos, J. Chem. Phys. 133, 124104 (2010).

[56] M. Ceriotti, G. Bussi, and M. Parrinello, Phys. Rev. Lett. 103, 30603 (2009).

[57] M. Ceriotti and D. E. Manolopoulos, Phys. Rev. Lett. 109, 100604 (2012).

[58] T. E. Markland and D. E. Manolopoulos, Chem. Phys. Lett. 464, 256 (2008).

[59] M. J. Van Vleet, A. J. Misquitta, A. J. Stone, and J. R. Schmidt, J. Chem. Theory Comput. 12, 3851 (2016).

[60] D. J. Cole, J. Z. Vilseck, J. Tirado-Rives, M. C. Payne, and W. L. Jorgensen, J. 
Chem. Theory Comput. 12, 2312 (2016).

[61] T. Bereau, R. A. DiStasio, A. Tkatchenko, and O. A. von Lilienfeld, J. Chem. Phys. 148, 241706 (2018), arXiv:1710.05871.

[62] A. P. Bartók, R. Kondor, and G. Csányi, Phys. Rev. B 87, 184115 (2013).

[63] P. Hohenberg and W. Kohn, Phys. Rev. 136, B864 (1964).

[64] W. Kohn and L. J. Sham, Phys. Rev. 140, A1133 (1965).

[65] W. L. Jorgensen and J. Tirado-Rives, J. Am. Chem. Soc. 110, 1657 (1988).

[66] W. D. Cornell, P. Cieplak, C. I. Bayly, I. R. Gould, K. M. Merz, D. M. Ferguson, D. C. Spellmeyer, T. Fox, J. W. Caldwell, and P. A. Kollman, J. Am. Chem. Soc. 117, 5179 (1995).

[67] S. J. Clark, M. D. Segall, C. J. Pickard, P. J. Hasnip, M. J. Probert, K. Refson, and M. C. Payne, Z. Krist. 220, 567 (2005).

[68] J. P. Perdew, K. Burke, and M. Ernzerhof, Phys. Rev. Lett. 77, 3865 (1996).

[69] C. Adamo and V. Barone, J. Chem. Phys. 110, 6158 (1999).

[70] F. L. Hirshfeld, Theor. Chim. Acta 44, 129 (1977).

[71] X. Chu and A. Dalgarno, J. Chem. Phys. 121, 4083 (2004).

[72] A. Ambrosetti, A. M. Reilly, R. A. DiStasio, and A. Tkatchenko, J. Chem. Phys. 140, 18A508 (2014).

[73] R. D. Goodwin and R. Prydz, J. Res. Natl. Bur. Stand. Sect. A Phys. Chem. 76A, $81(1972)$.

[74] Truncated to three significant digits; see reference for full precision.

[75] M. Ceriotti, G. Bussi, and M. Parrinello, J. Chem. Theory Comput. 6, 1170 (2010), arXiv:1204.0822v1.

[76] A. J. Russell and M. A. Spackman, Mol. Phys. 84, 1239 (1995).

[77] D. M. Bishop, F. L. Gu, and S. M. Cybulski, J. Chem. Phys. 109, 8407 (1998).

[78] A. S. Teja, R. J. Lee, D. Rosenthal, and M. Anselme, Fluid Phase Equilib. 56, 153 
(1990).

[79] D. J. C. MacKay, Information theory, inference, and learning algorithms (Cambridge University Press, Cambridge, 2003).

[80] C. E. Rasmussen and C. K. I. Williams, Gaussian Processes for Machine Learning (MIT Press, Cambridge, MA, 2006).

[81] A. Bartók-Pártay, L. Bartók-Pártay, F. Bianchini, A. Butenuth, M. Caccin, S. Cereda, G. Csányi, A. Comisso, T. Daff, S. John, C. Gattinoni, G. Moras, J. Kermode, L. Mones, A. Nichol, D. Packwood, L. Pastewka, G. Peralta, I. Solt, O. Strickson, W. Szlachta, C. Varnai, M. Veit, and S. Winfield, "libAtoms+QUIP," (2018), http://libatoms.org.

[82] M. Ceriotti, J. More, and D. E. Manolopoulos, Comput. Phys. Commun. 185, 1019 (2014).

[83] S. Plimpton, J. Comput. Phys. 117, 1 (1995).

[84] Using LAMMPS stable release from 11 Aug 2017.

[85] A. Jones and B. Leimkuhler, J. Chem. Phys. 135, 84125 (2011).

[86] D. Quigley and M. I. J. Probert, J. Chem. Phys. 120, 11432 (2004), arXiv:0403756 [cond-mat].

[87] L. Martínez, R. Andrade, E. G. Birgin, and J. M. Martínez, J. Comput. Chem. 30, 2157 (2009).

[88] A. Brünger, C. L. Brooks, and M. Karplus, Chem. Phys. Lett. 105, 495 (1984).

[89] S. Nosé, J. Chem. Phys. 81, 511 (1984).

[90] W. G. Hoover, Phys. Rev. A 31, 1695 (1985).

[91] M. Parrinello and A. Rahman, J. Appl. Phys. 52, 7182 (1981).

[92] W. Shinoda, M. Shiga, and M. Mikami, Phys. Rev. B 69, 134103 (2004).

[93] M. E. Tuckerman, J. Alejandre, R. López-Rendón, A. L. Jochim, and G. J. Martyna, J. Phys. A. Math. Gen. 39, 5629 (2006). 
[94] G. J. Martyna, D. J. Tobias, and M. L. Klein, J. Chem. Phys. 101, 4177 (1994).

[95] R. W. Hockney and J. W. Eastwood, Computer simulation using particles (Hilger, Bristol, 1988).

[96] T. B. Adler, G. Knizia, and H.-J. Werner, J. Chem. Phys. 127, 221106 (2007).

[97] G. Knizia, T. B. Adler, and H.-J. Werner, J. Chem. Phys. 130, 054104 (2009).

[98] L. Kong, F. A. Bischoff, and E. F. Valeev, Chem. Rev. 112, 75 (2012).

[99] S. Boys and F. Bernardi, Mol. Phys. 19, 553 (1970).

[100] H.-J. Werner, P. J. Knowles, G. Knizia, F. R. Manby, M. Schütz, P. Celani, T. Korona, R. Lindh, A. Mitrushenkov, G. Rauhut, K. R. Shamasundar, T. B. Adler, R. D. Amos, A. Bernhardsson, A. Berning, D. L. Cooper, M. J. O. Deegan, A. J. Dobbyn, F. Eckert, E. Goll, C. Hampel, A. Hesselmann, G. Hetzer, T. Hrenar, G. Jansen, C. Köppl, Y. Liu, A. W. Lloyd, R. A. Mata, A. J. May, S. J. McNicholas, W. Meyer, M. E. Mura, A. Nicklass, D. P. O’Neill, P. Palmieri, D. Peng, K. Pflüger, R. Pitzer, M. Reiher, T. Shiozaki, H. Stoll, A. J. Stone, R. Tarroni, T. Thorsteinsson, and M. Wang, "MOLPRO, version 2012.1, a package of ab initio programs," (2012).

[101] H.-J. Werner, P. J. Knowles, G. Knizia, F. R. Manby, and M. Schütz, WIREs Comput Mol Sci 2, 242 (2012).

[102] M. Schütz, R. Lindh, and H.-J. Werner, Mol. Phys. 96, 719 (1999).

[103] R. Lindh, Theor. Chim. Acta 85, 423 (1993).

[104] A. Hjorth Larsen, J. Jørgen Mortensen, J. Blomqvist, I. E. Castelli, R. Christensen, M. Dułak, J. Friis, M. N. Groves, B. Hammer, C. Hargus, E. D. Hermes, P. C. Jennings, P. Bjerre Jensen, J. Kermode, J. R. Kitchin, E. Leonhard Kolsbjerg, J. Kubal, K. Kaasbjerg, S. Lysgaard, J. Bergmann Maronsson, T. Maxson, T. Olsen, L. Pastewka, A. Peterson, C. Rostgaard, J. Schiøtz, O. Schütt, M. Strange, K. S. Thygesen, T. Vegge, L. Vilhelmsen, M. Walter, Z. Zeng, and K. W. Jacobsen, J. Phys. Condens. Matter 29, 273002 (2017). 
[105] R. M. Parrish, L. A. Burns, D. G. A. Smith, A. C. Simmonett, A. E. DePrince, E. G. Hohenstein, U. Bozkaya, A. Y. Sokolov, R. Di Remigio, R. M. Richard, J. F. Gonthier, A. M. James, H. R. McAlexander, A. Kumar, M. Saitow, X. Wang, B. P. Pritchard, P. Verma, H. F. Schaefer, K. Patkowski, R. A. King, E. F. Valeev, F. A. Evangelista, J. M. Turney, T. D. Crawford, and C. D. Sherrill, J. Chem. Theory Comput. 13, 3185 (2017).

[106] T. Verstraelen, P. Tecmer, F. Heidar-Zadeh, C. E. González-Espinoza, M. Chan, T. D. Kim, K. Boguslawski, S. Fias, S. Vandenbrande, D. Berrocal, and P. W. Ayers, "HORTON," (2017), http://theochem.github.com/horton/.

[107] A. D. Becke, J. Chem. Phys. 88, 2547 (1988).

[108] A. D. Becke and R. M. Dickson, J. Chem. Phys. 89, 2993 (1988).

[109] V. Lebedev and D. Laikov, Dokl. Math. 59, 477 (1999).

[110] J. D. Hunter, Comput. Sci. \& Eng. 9, 90 (2007).

[111] F. Pérez and B. E. Granger, Comput. Sci. Eng. 9, 21 (2007). 


\title{
Supporting information for: "Equation of state of fluid methane from first principles with machine learning potentials"
}

\author{
Max Veit* and Gábor Csányi \\ Engineering Laboratory \\ University of Cambridge \\ Trumpington Street \\ Cambridge, $C B 21 P Z$ \\ United Kingdom \\ Sandeep Kumar Jain, Satyanarayana Bonakala, and Indranil Rudra \\ Shell India Markets Pvt. Ltd. \\ Bengaluru 562149 \\ Karnataka, India \\ Detlef Hohl \\ Shell Global Solutions International BV \\ Grasweg 31 \\ 1031 HW Amsterdam \\ The Netherlands
}

(Dated: October 25, 2018) 


\section{DIMER ENERGIES}

The binding curves of the methane dimer shown in Figure 1 of the main paper were computed in a similar way as described in Gillan et. al. [1]: the Hartree-Fock (HF) energy was computed at the largest basis, the Dunning correlation-consistent basis set [2, 3] aug-ccpV5Z (hereafter called AV5Z). The energy difference between MP2 and HF was computed using the smaller AVQZ basis. Finally the difference between CCSD(T) (with explicitly correlated basis functions, called $\mathrm{CCSD}(\mathrm{T})-\mathrm{F} 12 \mathrm{a}[4,5])$ and MP2 was computed using the AVTZ basis. The corrections were successively added to the base HF energy to obtain energies at each of the HF, MP2, and CCSD(T)-F12 levels, and additionally forces at the HF and MP2 levels. Finally, all of the energies were corrected for basis-set superposition error (BSSE) using the Boys-Bernardi counterpoise procedure [6]. Calculations were done using the MOLPRO suite of programs [7].

The geometries for the symmetric orientations were generated using the Atomic Simulation Environment (ASE) [8] starting with monomers that had been optimized at the MP2/AVQZ level, resulting in a $\mathrm{C}-\mathrm{H}$ bond length of $1.085 \AA$. The first two configurations correspond exactly with configurations used in Chao et al. [9] and Hellmann et al. [10]; the figure gives the labels each author assigned to these configurations. The other three are similar, though not exactly the same, as the corresponding labeled configurations.

A first dimer model was obtained in a similar way as in Li and Chao [11], by fitting a pairwise L-J to the energies of the symmetric orientations shown in the main paper. The model was a standard 12-6 L-J between all atom pairs; the six coefficients for the different pair types were all optimized by a least-squares fit. The optimization produced $\mathrm{C}-\mathrm{H}$ and $\mathrm{H}-\mathrm{H}$ potentials that were nearly purely repulsive, so the form $\phi(r)=A r^{-12}$ was adopted for these instead. The C-C potential has the standard L-J form: $\phi(r)=-4 \epsilon\left((r / \sigma)^{-6}-(r / \sigma)^{-12}\right)$. The parameters of this model are given in Table I.

The fitting dataset used dimer distances from $3.5 \AA$ to $9.5 \AA$ in steps of $0.5 \AA$, with additional points at $3.25 \AA$ and $3.75 \AA$. Energies larger than $0.02 \mathrm{eV}$ were not used in the fit.

This model was then taken as the baseline, and further fits were done on the difference between this L-J baseline and the full energies in order to improve upon this model. For

* max.veit@epfl.ch; Current address: Laboratory of Computational Science and Modeling, Ecole Polytechnique Fédérale de Lausanne, 1015 Lausanne, Switzerland 


\begin{tabular}{ccc}
\hline \hline \multicolumn{2}{l}{ Parameter Pair type Value } \\
\hline$\sigma$ & C-C & $3.52608 \AA$ \\
$\epsilon$ & C-C & $0.00135 \mathrm{eV}$ \\
$A$ & C-H & $517.030 \mathrm{eV}$ \\
$A$ & H-H & $23.4878 \mathrm{eV}$ \\
\hline \hline
\end{tabular}

TABLE I. Parameters for the optimized pairwise L-J model

the new fits, a more thorough sample of the dimer configuration space was needed, so a random sample of dimers was taken from a liquid MD simulation using 200 rigid methane molecules with the monomer geometry optimized at the composite CCSD $(\mathrm{T}) /$ AVTZ level described below (but without the F12 correction), giving a C-H bond length of $1.088 \AA$, and the intermolecular interactions computed using the OPLS-AA force field [12]; the simulation was run using the LAMMPS molecular dynamics package $[13,14]$ at $188 \mathrm{~K}$ and 400 bar using a Langevin thermostat [15] and a Nosé-Hoover barostat[16-20]. (The MD simulations used to generate the random orientations for the binding curves in the main text were done the same way, except the monomers were fixed with the OPLS-AA C-H bond length of $1.09 \AA$.) The dimers were sampled with a C-C distance distribution from $3 \AA$ to $10 \AA$, strongly favoring the short range of $3 \AA$ to $5.5 \AA$ and further enriched between $3 \AA$ to $3.5 \AA$. A section of this distribution is pictured with the dimer binding curves in the main text; the full sample contains 2418 dimers. The interaction energies of the dimers in this dataset were computed using the same procedure as described for the fixed-orientation samples.

We first fit to MP2, since both energies and forces are available to achieve a high-quality fit. The simplest descriptor used was the distance between pairs of atoms; each type of pair (e.g. C-C, C-H, and H-H for methane) is given a separate Gaussian process corresponding to a separate pair potential. This descriptor is called '2b' (for "atomwise two-body"). This idea can be extended to triplets of atoms, where the set of three distances is symmetrized so as to make it permutationally invariant. This descriptor is likewise called ' $3 \mathrm{~b}$ '. A first fit was done using both of the above descriptors, with one Gaussian process for each pair or triplet type; the resulting potential is essentially a sum of atomwise pair and triplet potentials with fully flexible functional forms. This potential is called the ' $2 \mathrm{~b}+3 \mathrm{~b}$ GAP'. As Figure 1 shows, though, this fit offers only a modest improvement over the baseline. 



FIG. 1. Errors of successive GAP models fit to MP2 data, shown as a function of C-C dimer separation. The baseline is a pairwise L-J model fitted to the coupled-cluster data from the symmetric orientations. The first fit uses two-body and three-body descriptors, the second uses the 6-D dimer descriptor, and the final correction to the coupled-cluster level is a simple two-body (pairwise) fit.

We therefore attempt a fit in the full six-dimensional space of rigid dimer configurations using the dimer descriptor. This descriptor is composed of the set of distances between all atom pairs in the dimer, symmetrized over permutations of like atoms. Concretely, the kernel or covariance function between two dimers is, as described in [21]:

$$
k\left(\mathbf{R}, \mathbf{R}^{\prime}\right)=\delta \exp \left[-\sum_{i} \frac{\left(R_{i}-R_{i}^{\prime}\right)^{2}}{\left(2 \sigma_{i}^{2}\right)}\right],
$$

where $\mathbf{R}$ is the set of distances between all atoms in the dimer, $\delta$ is the characteristic energy scale of variation of the function, and the $\sigma_{i}$ are the charateristic length scales for each distance type. This kernel must be permuationally symmetrized so that the resulting potential does not depend on the order of the atoms:

$$
\tilde{k}\left(\mathbf{R}, \mathbf{R}^{\prime}\right)=\frac{1}{|S|} \sum_{\pi \in S} k\left(\pi(\mathbf{R}), \mathbf{R}^{\prime}\right)
$$


where $S$ is the permutation group of the methane dimer, which - allowing both swaps of hydrogen atoms within the monomers and swaps of whole monomers in the dimer - has order $4 ! \times 4 ! \times 2=1152$. The kernel is finally multiplied by a cutoff function $f_{\text {cut }}\left(r_{a b}\right) f_{\text {cut }}\left(r_{a b}^{\prime}\right)$, one for each dimer, which depends on the center-of-mass separation of the monomers in the dimer. The cutoff function is designed to take the function smoothly to zero as either of the dimers approaches some cutoff distance; in our implementation, it takes the form of a half-cosine between an inner and an outer cutoff; the functional form is given in [22].

It is an overcomplete representation of the full space of mutual dimer orientations, which in the case of rigid methanes is six-dimensional. The resulting fit offers improvements of at least an order of magnitude across the entire close range ( $3 \AA$ to $6 \AA$ ). The final correction is the difference from MP2 to coupled cluster $\operatorname{CCSD}(\mathrm{T})-\mathrm{F} 12$, which is easily captured to high accuracy using an atomwise two-body (pairwise) GAP fit to the original sample of 896 dimers (a subset of the full sample, without the subsequent short-range augmentation needed for the MP2 dimer GAP). The composite model created by adding the L-J baseline, the MP2 dimer GAP, and the final coupled-cluster two-body GAP, will hereafter be referred to as the '6-D dimer GAP'. The resulting potential is a pairwise-additive two-body model and will thus miss all beyond-two-body (beyond-dimer) effects. It will still serve as a useful reference for further models, though, as it can be taken as the benchmark standard for the fictitious system of methane with only two-body interactions present.

The new potential was evaluated on its own training set and on the dimer binding curves from the main text. It consistently achieves the level of accuracy specified in the fit, $2 \mathrm{meV}$, in the regions of the potential probed under liquid conditions (as evidenced by the pair correlation function) and can therefore be used as a reference standard for liquid methane dimer interactions.

\section{GAP FITS AND EVALUATION}

GAP is a statistical learning method and hence the quality of the SOAP fits can be evaluated by how well they reproduce the energies and forces of the training set. RMS energy and force errors are given in Table II, with the 6-D dimer GAP errors given for comparison. 


\begin{tabular}{lcc}
\hline \hline GAP name & RMS energy error $/\left(\mu \mathrm{eV} / \mathrm{CH}_{4}\right)$ & $\mathrm{RMS}$ force error $/(\mathrm{meV} / \AA)$ \\
\hline PBE SOAP-GAP & 200 & 7.36 \\
PBE0 SOAP-GAP & 207 & 6.58 \\
MBD(PBE) SOAP-GAP & 76.1 & $1.3 \quad(\mathrm{FD})$ \\
MBD(PBE0) SOAP-GAP & 76.9 & - \\
MP2 dimer GAP & 367 & -10 \\
CCSD(T)-F12 2b GAP & 80.6 & - \\
6-D dimer GAP & 381 & - \\
\hline \hline
\end{tabular}

TABLE II. RMS energy and force training errors of the GAP fits

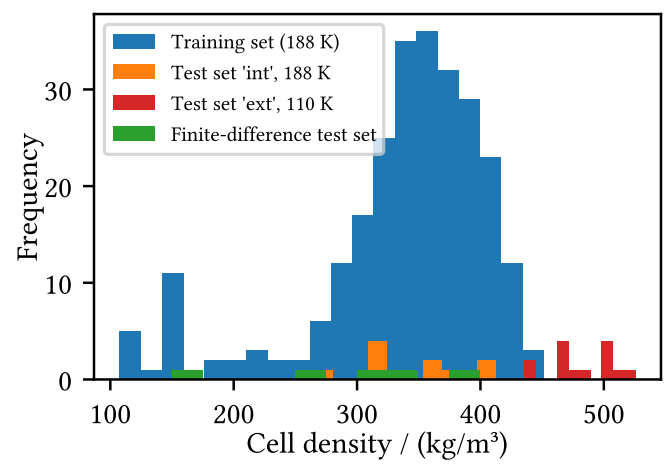

FIG. 2. Histogram of densities in the training set and the three test sets: Interpolation, extrapolation, and the five finite-difference geometries.

The fits are additionally evaluated on two test sets that were not included in the training set, as described in the main text. Additionally, the performance of the MBD SOAP-GAP fits were assessed by the finite-difference method, as gradients were not available. For this purpose, a sample of five small cells containing eight methane molecules each was taken from OPLS/AMBER NVT simulations, one each at five densities ranging from $150 \mathrm{~kg} / \mathrm{m}^{3}$ to $400 \mathrm{~kg} / \mathrm{m}^{3}$ (see Figure 2). Each geometry was displaced in each of five randomly selected directions for a total of 25 finite-difference forces. The results are shown in the right-hand panels of Figure 4.

The parameters for the above fits are given as command lines that can be used with 


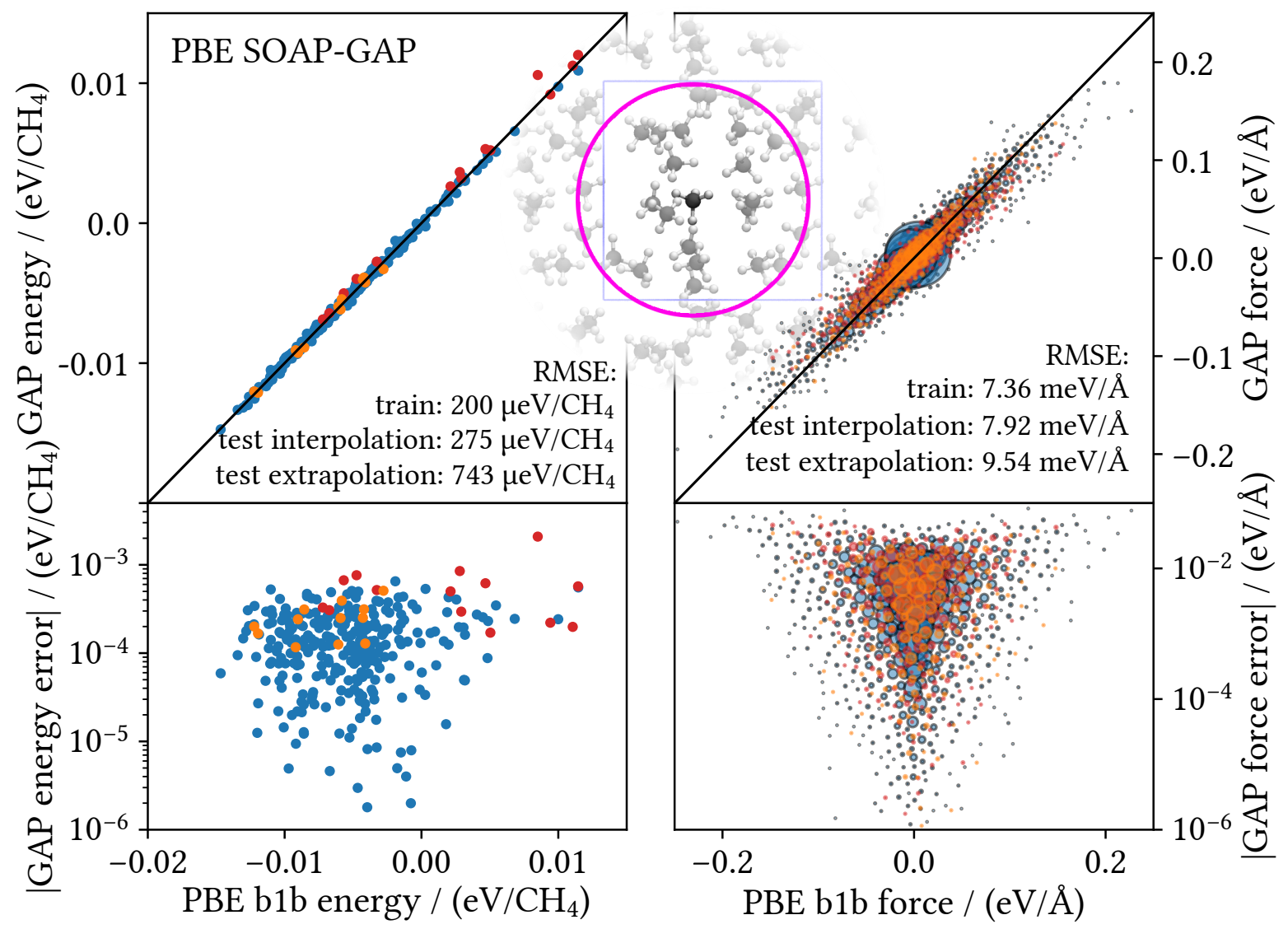

FIG. 3. The PBE SOAP-GAP fit on 277 cell interaction (beyond one-body, 'b1b') energies and corresponding forces. Left: energies; right: Cartesian force components. In the force plots, due to the large number of points, only a subset of representative points are shown with their sizes scaled according to the 0.8 power of the number of points they represent. Top: Correlation plots with the line $y=x$ of perfect correlation. Bottom: Errors on a logarithmic scale. The blue points represent the training set. The orange points represent the interpolation test set and the red points represent the extrapolation test set (color online), neither of which was used in training the model. 


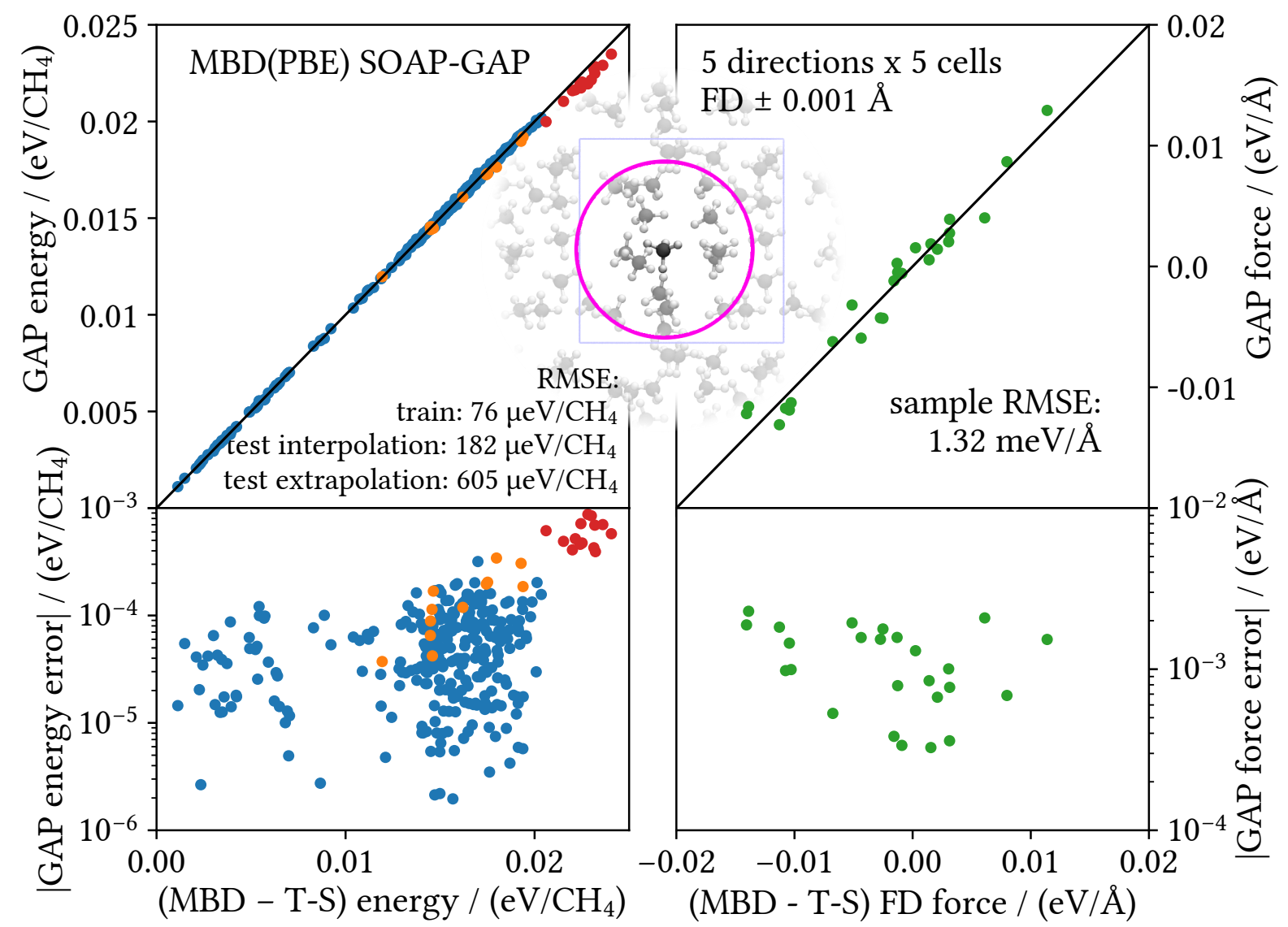

FIG. 4. The MBD(PBE) SOAP-GAP fit to the differences between the MBD interaction energy and the T-S interaction energy, both computed using PBE-derived Hirshfeld volumes (fixed averages for T-S). Left: Energies; blue points for the training set, orange points for the interpolation test set and red points for the extrapolation test set (color online). Right: Forces estimated by finite differences on five cells of eight methanes each in five randomly chosen directions each (green points). As before, correlation plots against $y=x$ above, log errors below. 
the 'teach_sparse' command in the libAtoms/QUIP package [23]. The GAP code can be downloaded at http://www.libatoms.org/gap/gap_download.html, with a prepackaged version available through Docker at https://hub.docker.com/r/libatomsquip/quip/.

The parameters for the PBE SOAP-GAP are (all on one line):



(The parameters for the PBE0 SOAP-GAP are exactly the same; only the source data was computed with PBE0 instead of PBE)

The parameters for the MBD SOAP-GAP are (again, same for both PBE and PBE0 energies):

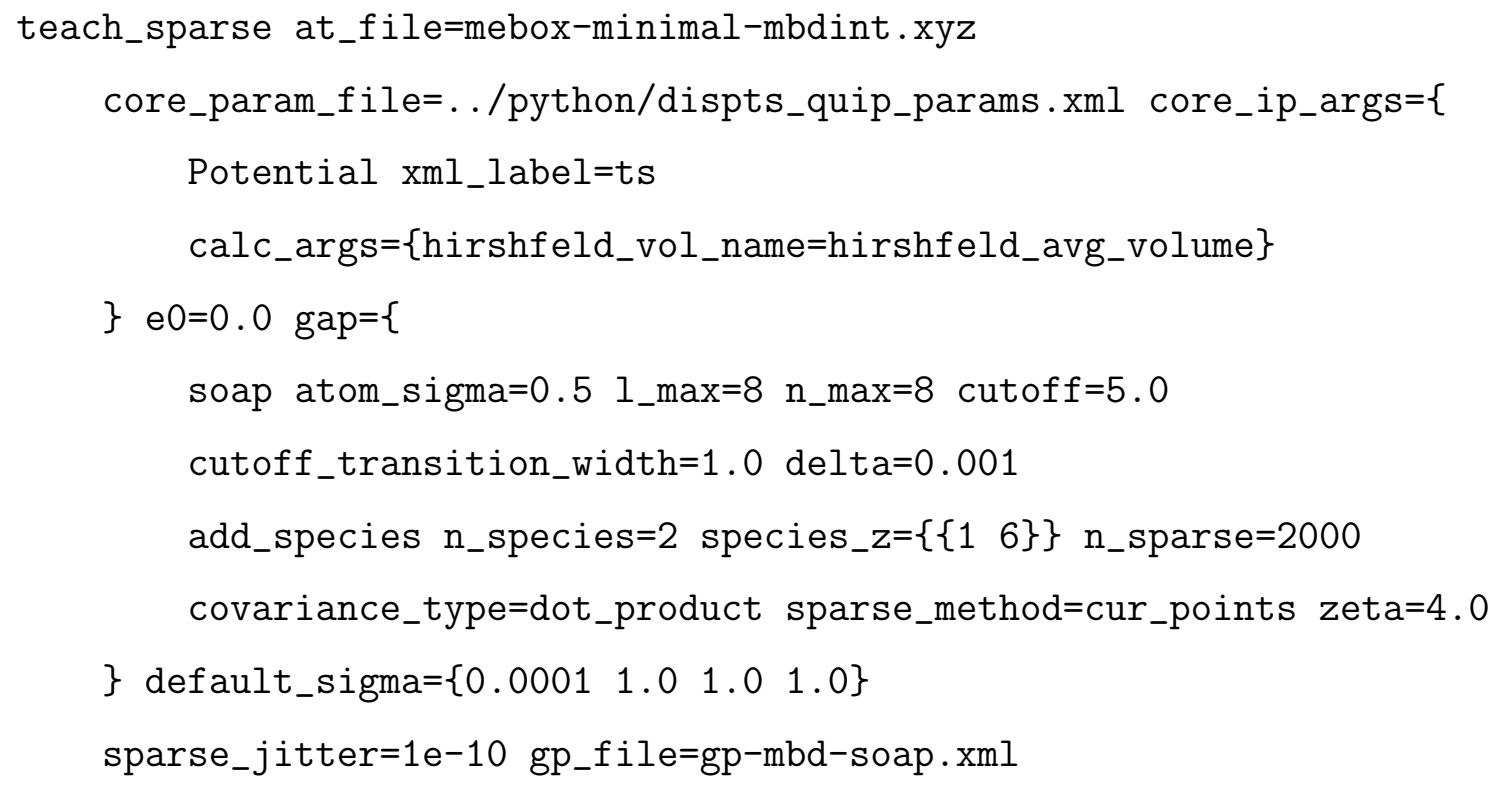


The parameters for the 6-D dimer fit to MP2 are:

$$
\begin{aligned}
& \text { teach_sparse at_file=me-rigid-shortaug3-mp2-avqz-intnonan.xyz } \\
& \text { core_param_file }=\{. \text { /empirical-pots/ljrep_quip_params.xml }\} \\
& \text { core_ip_args }=\{\text { IP LJ }\} \text { gap }=\{ \\
& \text { general_dimer cutoff }=6.0 \text { cutoff_transition_width }=1.0 \\
& \text { signature_one } \left.=\left\{\begin{array}{lllll}
6 & 1 & 1 & 1 & 1
\end{array}\right\} \text { signature_two }=\left\{\begin{array}{lllll}
6 & 1 & 1 & 1 & 1
\end{array}\right\}\right\} \\
& \text { monomer_one_cutoff=1.5 monomer_two_cutoff=1.5 atom_ordercheck=F } \\
& \text { strict }=F \text { mpifind=T theta_uniform=1.0 covariance_type=ARD_SE } \\
& \text { n_sparse }=2000 \text { delta=0.02 sparse_method=CUR_COVARIANCE } \\
& \} \text { default_sigma }=\{0.00020 .0020 .00 .0\} \text { sparse_jitter }=10-10 \\
& \text { energy_parameter_name=energy force_parameter_name=force e } 0=0.0 \\
& \text { gp_file=gp-merig-mp2-gendim-shortaug3.xml do_copy_at_file=F }
\end{aligned}
$$

And for the much simpler two-body (atomwise) fit to the CCSD(T)-MP2 difference:

$$
\begin{aligned}
& \text { teach_sparse at_file=me-rigid-train-ljrep.xyz gap= } \\
& \text { distance_2b cutoff=10.0 covariance_type=ARD_SE } \\
& \text { n_sparse=50 sparse_method=UNIFORM } \mathrm{Z1=6} \mathrm{Z} 2=6 \\
& \text { theta_fac=0.2 delta=0.0005 resid_name=resid only_inter=T } \\
& \text { : distance_2b cutoff=6.0 covariance_type=ARD_SE } \\
& \text { n_sparseX=50 sparse_method=uniform } \mathrm{Z1=1} \mathrm{Z2=6} \\
& \text { theta_fac=0.2 delta=0.0005 resid_name=resid only_inter=T } \\
& \text { : distance_2b cutoff=6.0 covariance_type=ARD_SE } \\
& \text { n_sparseX=50 sparse_method=uniform Z1=1 Z2=1 } \\
& \text { theta_fac=0.2 delta=0.0005 resid_name=resid only_inter=T } \\
& \text { \} default_sigma=\{0.00001 } 0.0 \text { } 0.0\} \text { sparse_jitter=1e-10 } \\
& \text { energy_parameter_name=ediff_cc force_parameter_name=none e0=0.0 } \\
& \text { gp_file=gp-merig-cc-ljrep-2b.xml do_copy_at_file=F sparse_separate_file=F }
\end{aligned}
$$

The final 6-D dimer GAP is simply the sum of the above two potentials.

These potentials, once fitted, are stored in the form of an XML file that can be read by QUIP to evaluate energies and forces on any new configuration. The XML files for the above GAPs are avalable online in the Apollo repository [24] as well as on our group's webpage [25]. 


\section{DFT AND MBD PARAMETERS}

As mentioned in the main text, the sample for the DFT calculations was taken from MD trajectories under liquid conditions run using a classical potential (OPLS/AMBER [26, 27]) at a temperature of $188 \mathrm{~K}$ and five pressures ranging from 0 bar to 400 bar (the same ones at which OPLS/AMBER was tested in the main text, with the addition of 0 bar). There were 60 samples taken from each pressure, with the exception of the shorter 0 bar simulation, which only contributed 40 samples. Each of the 280 cells in the sample contained 27 (flexible) methane molecules; otherwise, the simulation parameters were the same as those described later in Section IV B.

The DFT calculations were all done with the CASTEP code [28], version 8.0. The PBE calculations were done with a plane-wave cutoff of $650 \mathrm{eV}$ and the default finite-basis correction. Due to the large, amorphous nature of the system, no k-point sampling was employed; calculations were only done at the $\Gamma$ point. Convergence tolerances were set to $1 \mu \mathrm{eV} / \mathrm{atom}$ for the energies and $10 \mu \mathrm{eV} / \AA$ for the forces. The PBE0 calculations were done with a cutoff of $700 \mathrm{eV}$ and no finite-basis correction, again only at the $\Gamma$ point, and convergence tolerances one order of magnitude smaller $(0.1 \mu \mathrm{eV} /$ atom for the energies and $1 \mu \mathrm{eV} / \AA$ for the forces). Since computing the interaction energy requires subtracting the one-body contribution (the energy and force of each individual methane molecule in the cell) and the samples had flexible monomer geometries, an additional calculation was run on each of the 27 individual molecules in each cell, using the same periodic boundary conditions as the full cell. The energy that resulted from subtracting the sum of the monomer energies from the total cell energy is the interaction or beyond-one-body ('b1b') energy (and likewise with the interaction force). Finally, two cells were discarded because their interaction energies (both PBE and PBE0) were much higher than the rest; those cells came from the initial MD equilibration from a high-energy geometry, so they were removed to achieve a better fit for normal, equilibrium conditions. Additionally, the largest cell for PBE and the largest 20 cells for PBE0 did not complete because the computational requirements exceeded available resources. The training set therefore comprised $277 \mathrm{PBE}$ interaction energies (and $277 \times 135 \times 3=112185$ PBE force components), and 258 PBE0 interaction energies (and $258 \times 135 \times 3=104490$ PBE0 force components). These sets of interaction energies and forces were finally fit with the SOAP GAPs above, ranged at $6 \AA$. 
The MBD energies were computed on the same sets of 277 (or 258 for PBE0) methane cells using the implementation available at http://www.fhi-berlin.mpg.de/ tkatchen/MBD/ and interfaced with QUIP. This was done both with the PBE and PBE0 Hirshfeld volumes calculated from each geometry, as reported by CASTEP. The supercell cutoff parameter was adjusted so that a $1 \times 1 \times 1$ supercell (that is, only the unit cell) was used, in correspondence with the omission of k-point sampling in the DFT calculations. All other MBD paramters were left at their defaults. The corresponding T-S model, with fixed, per-element averaged PBE or PBE0 volumes, was then subtracted and the difference was fit with a SOAP-GAP ranged at $5 \AA$. The magnitude of the correction beyond this range was small enough that neglecting it was seen as safe. The given implementation did not implement gradients, so the accuracy of the GAP forces was assessed using a finite-difference scheme as described above.

The Hirshfeld volumes used to compute T-S and MBD energies on the dimer test set (computed to assess PBE+MBD and PBE0+MBD dimer model errors) were instead computed from the wavefunctions produced by the PSI4 code [29] with the HORTON post-processing functionality [30], which itself uses methods derived by Becke and Dickson for polyatomic molecules [31-33].

\section{MD PARAMETERS}

\section{A. GAP fits}

Most of the GAP MD simulations were run with i-PI [34], interfaced through LAMMPS [13, 35] to QUIP [23]. Only the 6-D dimer GAP simulation was run with QUIP's built-in MD functionality. It used the adaptive Langevin thermostat of Jones and Leimkuhler [36] (with a time constant of $10 \mathrm{fs}$ ) and a Hoover-Langevin barostat [37] (with a time constant of $100 \mathrm{fs}$ and a mass factor of 100). For the SOAP simulations, the T-S correction was cut off at $15 \AA$, smoothed with a half-cosine curve over $1 \AA$. Likewise, the L-J baseline (as a component of the general dimer GAP) was cut off at $15 \AA$ and smoothed over $1 \AA$ (C-C potential only; the other two were simply cut off at $10 \AA$ ). Analytical tail corrections were calculated by 
(PBE0 SOAP)/COMPASS + T-S + MBD(PBE0) SOAP:

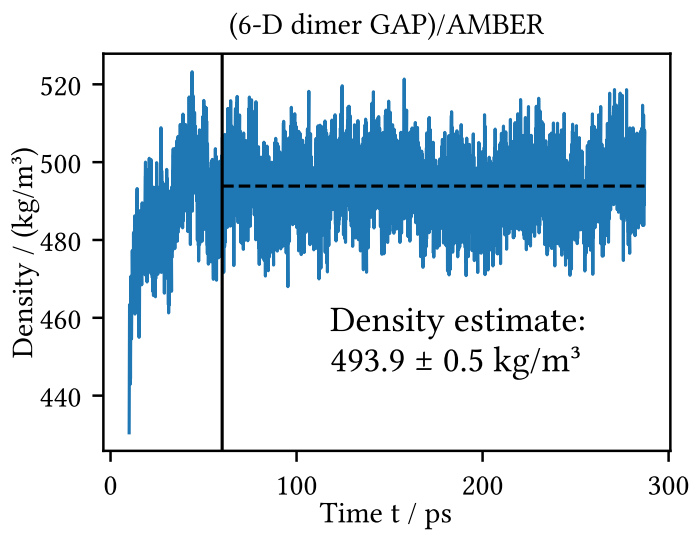

PIMD $188 \mathrm{~K}, 278$ bar
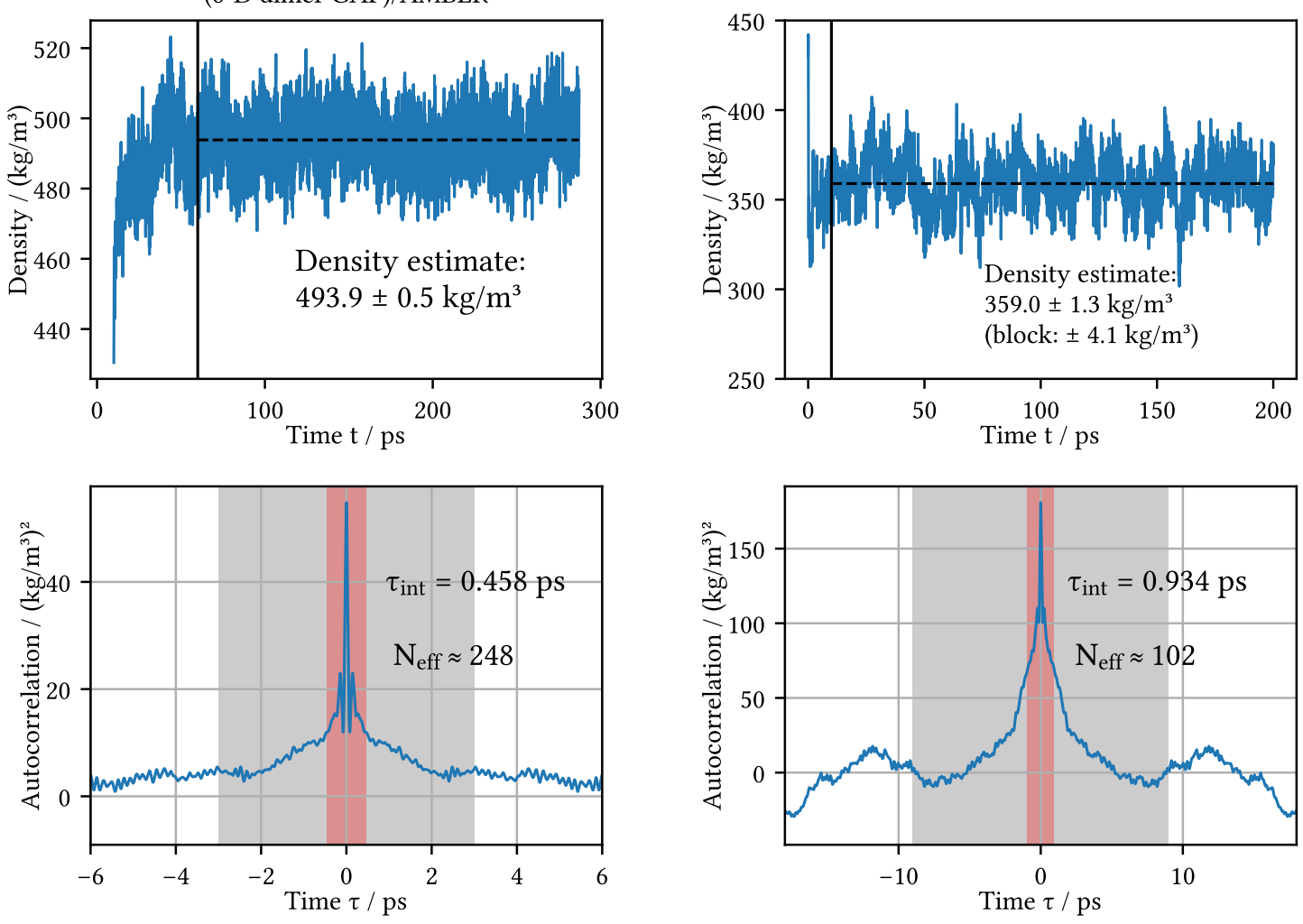

FIG. 5. Trace of the density of the GAP NPT simulations over time. Averaging was done starting from the vertical solid line. The autocorrelation of the density timeseries (discarding the initial transient) is shown in the bottom plots. The integral $\tau_{\text {int }}$ of the normalized autocorrelation is a good estimate for the series's correlation time, which in turn can be used to estimate the number of effective independent samples $N_{\text {eff }}=\frac{T}{2 \tau_{\text {int }}}$ ( $T$ is the length of the series being averaged) and the standard error on the mean $\sigma_{\text {corr }}=\sqrt{\frac{\sigma_{0}^{2}}{2 N_{\text {eff }}}}$ (where $\sigma_{0}^{2}$ is the variance of the sample being averaged). The grey region is the integration region and the red shows the correlation time. 
(PBE0 SOAP)/COMPASS + T-S + MBD(PBE0) SOAP:

PIMD $188 \mathrm{~K}, 163$ bar
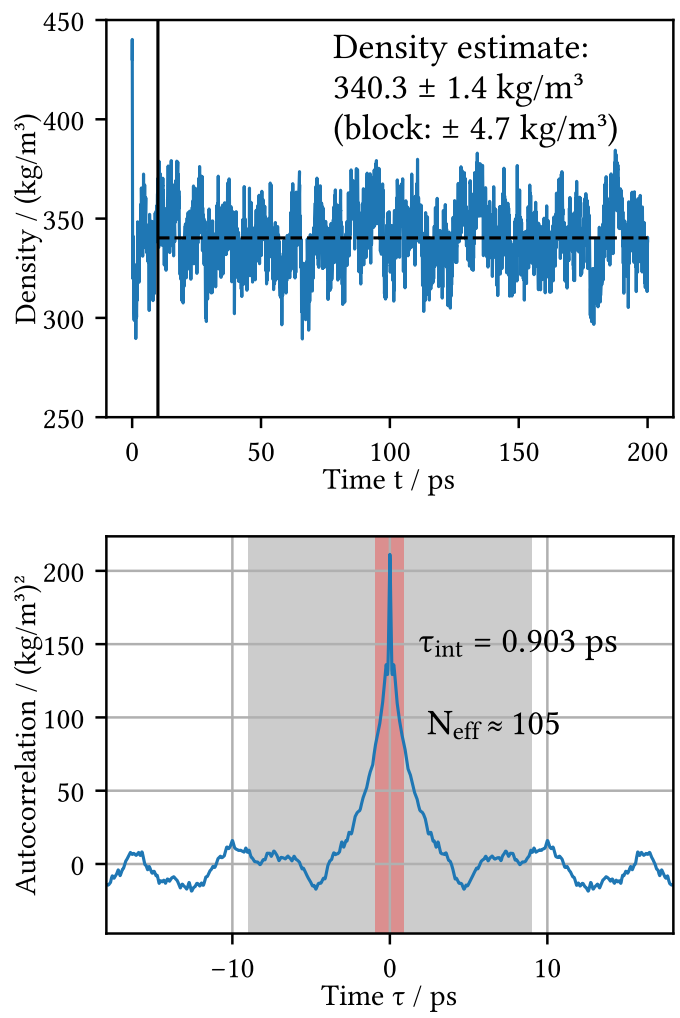

(PBE0 SOAP)/COMPASS + T-S + MBD(PBE0) SOAP: PIMD $188 \mathrm{~K}, 86.9$ bar
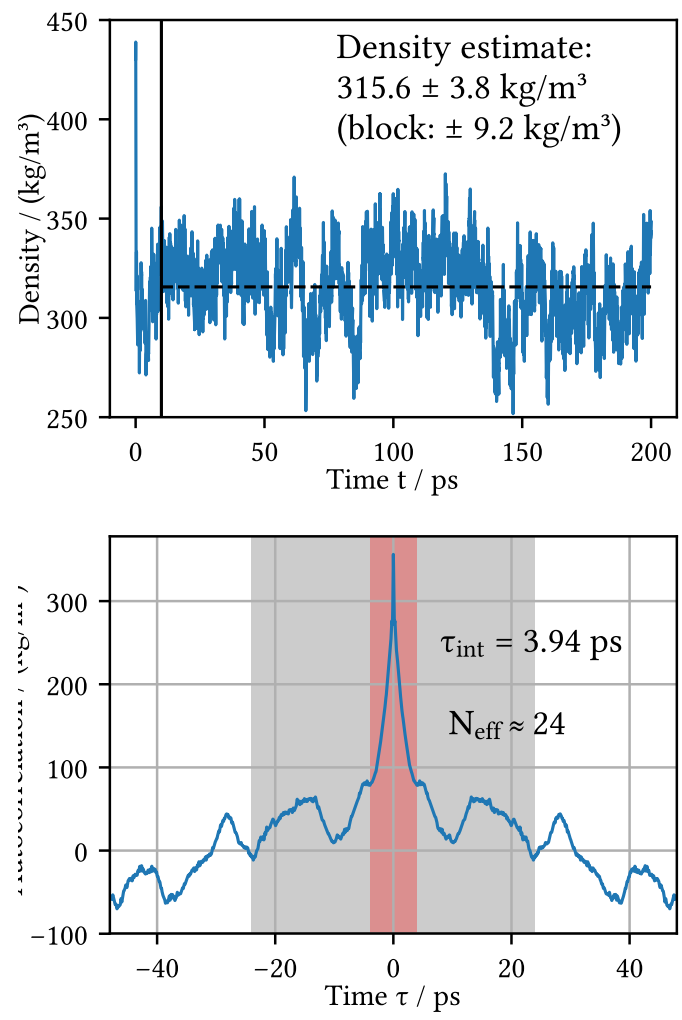

FIG. 6. Trace of the density of the GAP NPT simulations over time: (PBE0 SOAP)/COMPASS $+\mathrm{T}-\mathrm{S}+\mathrm{MBD}(\mathrm{PBE} 0) \mathrm{SOAP}$ at $188 \mathrm{~K}$. As in Figure 5, timeseries at the top, autocorrelation plots at the bottom. Most of the PIMD simulations are not yet completely equilibrated (as can be seen both in the time trace and the autocorrelation function), so an interim estimate was also computed by splitting the utilized simulation time into 10 blocks and taking the standard deviation of the individual means of the blocks. 
(PBE0 SOAP)/COMPASS + T-S + MBD(PBE0) SOAP: PIMD 110 K, 316 bar


(PBE0 SOAP)/COMPASS + T-S + MBD(PBE0) SOAP: PIMD $110 \mathrm{~K}, 238$ bar
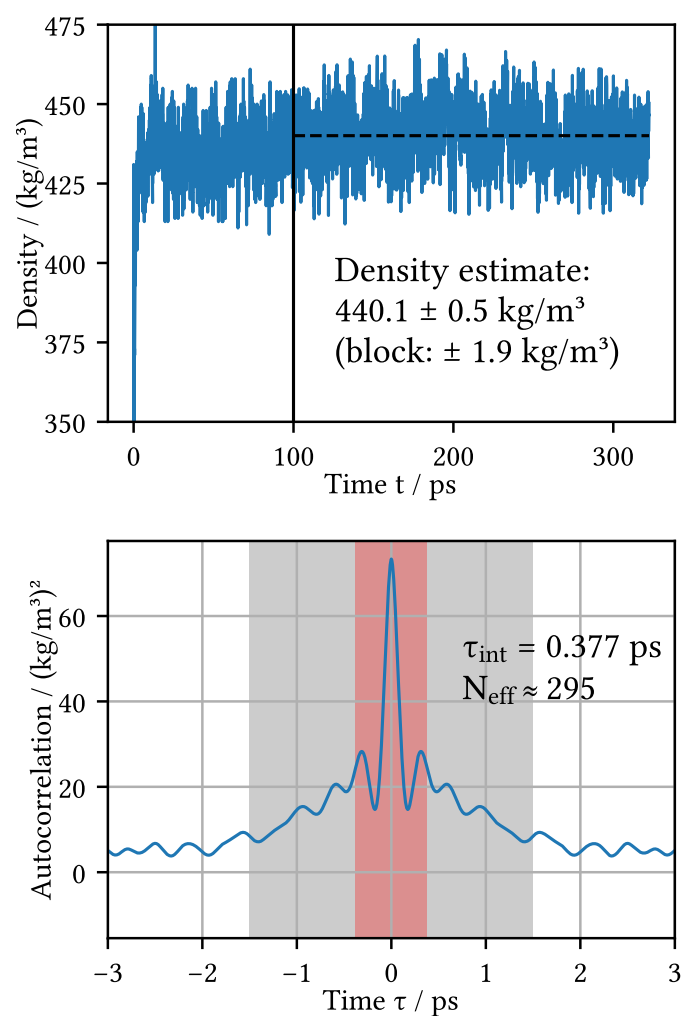

FIG. 7. Trace of the density of the GAP NPT simulations over time: (PBE0 SOAP)/COMPASS $+\mathrm{T}-\mathrm{S}+\mathrm{MBD}(\mathrm{PBE} 0) \mathrm{SOAP}$ at $110 \mathrm{~K}$. As in Figure 5, timeseries at the top, autocorrelation plots at the bottom.

computing the integral of the missing energy and virial outside the inner cutoff of $14 \AA$; see Section V for details. The initial configuration for these simulations was a 100-methane cell generated using Packmol [38].

The i-PI simulations used a thermostat based on the generalized Langevin equation (GLE, otherwise known as colored-noise thermostats), namely the "smart sampling" method of Ceriotti, Bussi, and Parrinello [39]. The parameters were generated at http://gle4md.org/ using the parameters $t_{\mathrm{opt}}=10 \mathrm{ps}, N_{s}=6$, and $\omega_{\max } / \omega_{\min }=10^{4}$ for the thermostat and $t_{\text {opt }}=2 \mathrm{ps}, N_{s}=6$, and $\omega_{\max } / \omega_{\min }=10^{3}$, and a piston time constant of $\tau=100$ fs for the barostat.

The PIMD simulations used the PIGLET $[40,41]$ thermostat to accelerate convergence 
(PBE0 SOAP)/COMPASS + T-S + MBD(PBE0) SOAP: PIMD $110 \mathrm{~K}, 178$ bar
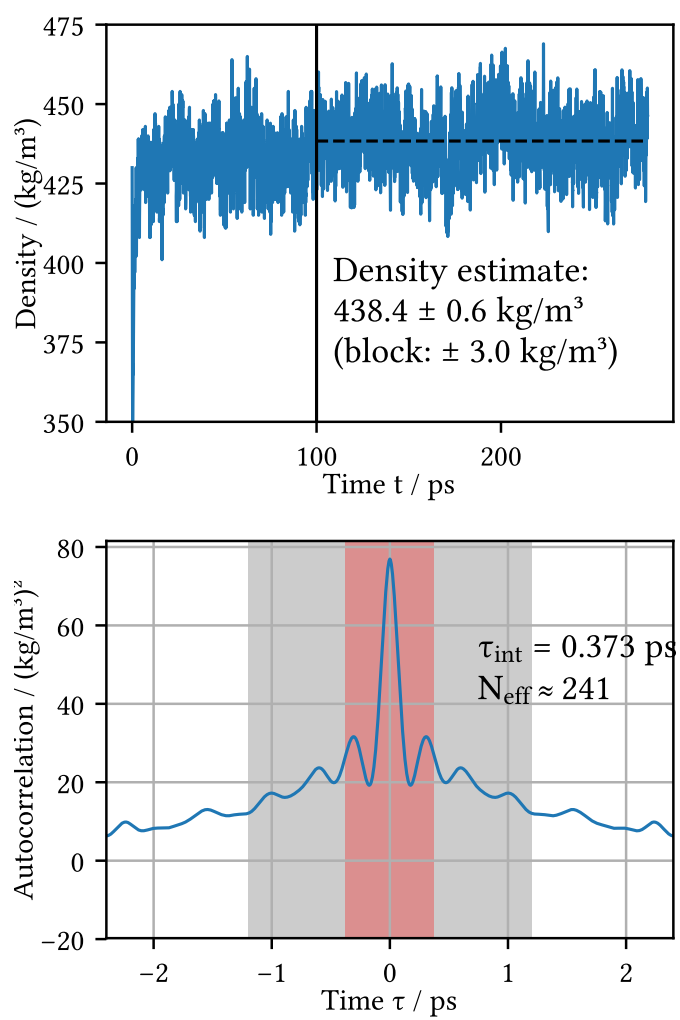

(PBE0 SOAP)/COMPASS + T-S + MBD(PBE0) SOAP: PIMD $110 \mathrm{~K}, 116$ bar


FIG. 8. Trace of the density of the GAP NPT simulations over time: (PBE0 SOAP)/COMPASS $+\mathrm{T}-\mathrm{S}+\mathrm{MBD}(\mathrm{PBE} 0) \mathrm{SOAP}$ at $110 \mathrm{~K}$. As in Figure 5, timeseries at the top, autocorrelation plots at the bottom.

to the quantum partition function. The thermostat parameters were generated at the same website, this time using the PIGLET paramters of $\mathrm{OPT}(\mathrm{H}), N_{s}=8, \omega_{\max }=3000 \mathrm{~cm}^{-1}$, $\omega_{\max } / \omega_{\min }=10^{4}, \hbar \omega / k_{B} T=50$, with the appropriate temperature $T(110 \mathrm{~K}$ or $188 \mathrm{~K})$, and with both 12 and 16 beads to verify convergence to the quantum limit; the larger number was used in production simulations. The centroid barostat used the analogous "optimal sampling" method $[39,42]$ with the same parameters: Potential energy optimized, $N_{s}=8$, $\omega_{0}=30 \mathrm{~cm}^{-1}$, and $\omega_{\max } / \omega_{\min }=10^{4}$ (resulting in $\left.\omega_{\max }=3000 \mathrm{~cm}^{-1}\right)$.

The parameter files for the above thermostats, including the matrices used to propagate the generalized Langevin equation, are available in the Apollo repository [24] as well as on our group's webpage [25]. 
(PBE0 SOAP)/COMPASS + T-S + MBD(PBE0) SOAP:

PIMD $110 \mathrm{~K}, 64.5$ bar
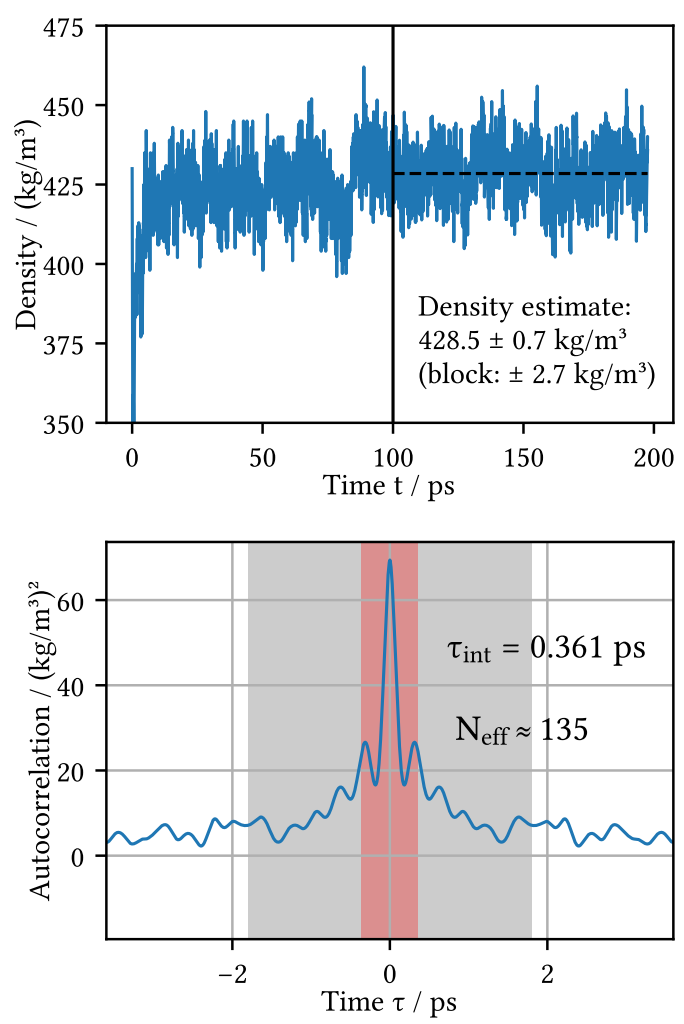

(PBE0 SOAP)/COMPASS + T-S + MBD(PBE0) SOAP: PIMD $110 \mathrm{~K}, 5.93$ bar
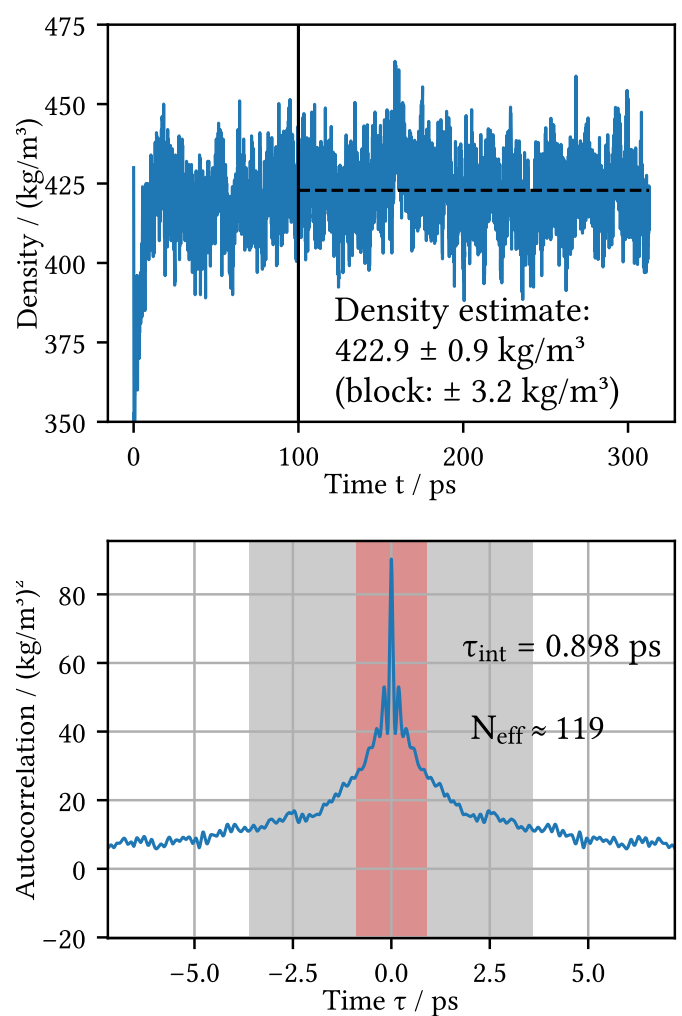

FIG. 9. Trace of the density of the GAP NPT simulations over time: (PBE0 SOAP)/COMPASS $+\mathrm{T}-\mathrm{S}+\mathrm{MBD}(\mathrm{PBE} 0) \mathrm{SOAP}$ at $110 \mathrm{~K}$. As in Figure 5, timeseries at the top, autocorrelation plots at the bottom.

No smooth cutoffs were done in the i-PI simulations due to the necessity of interfacing with QUIP through LAMMPS; analytical tail corrections were applied, though. The initial configuration was prepared with an initial 10 ps NVT equilibration using the '(PBE0 SOAP)/COMPASS + T-S + MBDGAP' potential; this configuration was used for both the classical and PIMD simulations at that temperature. All GAP MD simulations were done with a timestep of $0.5 \mathrm{fs}$.

Each run had a certain amount of initial equilibration time discarded from its trajectory, depending chiefly on the potential, the thermostat, and the temperature. The ten pairs of plots in Figures 5 through 9 show how the average density and standard error were obtained from the time evolution of the density for the 6-D dimer GAP simulation and 
for the '(PBE0 SOAP)/COMPASS + T-S + MBD(PBE0) SOAP' PIMD simulations. The standard error was obtained by integrating the autocorrelation of the density timeseries as described in Sokal [43]. However, many simulations showed extremely long correlation times and were not fully equilibrated within the available simulation time, rendering the autocorrelation method inapplicable. Therefore, to estimate the error incurred due to the large-scale fluctuations still observed, the simulation time utilized for averaging was split into ten equal blocks (corresponding approximately to the timescale of fluctuations still observed), the mean value was computed within each of those blocks, and the final error estimate computed as the standard deviation of those means. These error estimates are displayed as error bars on the '(PBE0 SOAP)/COMPASS + T-S + MBD(PBE0) SOAP' PIMD simulation results in Figure 5 of the main text.

\section{B. Analytical potentials}

The analytical potentials were run in LAMMPS [13, 14] with a Langevin thermostat [15] and a Nosé-Hoover barostat [16-20] with the MTK correction [44], both using a time constant of $0.1 \mathrm{ps}$, and an initial configuration of 200 methane molecules generated using Packmol [38] and relaxed with the OPLS-AA [12] forcefield. All simulations used analytical tail corrections to account for the otherwise-neglected dispersion energy beyond their cutoffs [45, 46] (for the L-J baseline this was only done for the C-C potential). For potentials with a Coulomb component (OPLS/AMBER and COMPASS), the contributions beyond the cutoff were calculated with the particle-particle particle-mesh (PPPM) method [47]. The MD timesteps were $1 \mathrm{fs}$ for TraPPE, $0.5 \mathrm{fs}$ for the Li-Chao L-Jand OPLS/AMBER at $110 \mathrm{~K}$, and $0.1 \mathrm{fs}$ for the others (OPLS/AMBER at $188 \mathrm{~K}$, the L-J baseline, and COMPASS).

The potentials themselves used L-J cutoffs (and Coulomb cutoffs for OPLS/AMBER and COMPASS) of $10 \AA$, except for TraPPE, where the cutoff of $14 \AA$ recommended on the website was used instead. The two pairwise L-J fits (the L-J baseline and Li-Chao) both were added to the AMBER intramolecular terms to give complete liquid methane potentials.

Equilibration and run times again varied between the potentials based on the rate of convergence of the density, although the same times were used throughout an isotherm. The times are summarized in Table III. 


\begin{tabular}{rccc}
\hline Potential & Temperature/K Equilib. time/ps Run time/ps \\
\hline TraPPE & 110 & 100 & 400 \\
& 188 & 100 & 400 \\
OPLS/AMBER & 110 & 25 & 75 \\
& 188 & 100 & 400 \\
L-J baseline & 110 & 50 & 50 \\
& 188 & 300 & 200 \\
Li-Chao L-J & 110 & 100 & 400 \\
\hline \hline
\end{tabular}

TABLE III. Equilibration and run times for the analytical potentials

\section{TAIL CORRECTIONS WITH A SMOOTH CUTOFF}

For all the NPT simulations done for this work it was found important to incorporate tail corrections to account for the missing pressure neglected by cutting off the long-range dispersion potentials (the sixth-power part of L-J and T-S). These corrections can also be applied in the case where the potential is smoothed to zero before the cutoff, though the resulting integrals become more difficult to evaluate.

The expression for the missing pressure in a potential that is cut off with a smoothing function that starts at $r_{\text {in }}$ and ends at $r_{\text {out }}$ is, by straightforward extension of the formulae in $[45,46]$ :

$$
\begin{aligned}
p_{\text {exact }}-p_{\text {cut }}=\frac{1}{6} \sum_{i=1}^{n_{\text {typ }}} \rho_{i} \sum_{j=1}^{n_{\text {typ }}} \rho_{j} & \int_{r_{\text {in }}}^{\infty} r \frac{\mathrm{d}}{\mathrm{d} r}\left(\phi_{i j}(r)-\phi_{i j, \text { cut }}(r)\right) 4 \pi r^{2} g_{i j}(r) \mathrm{d} r \\
=\frac{1}{6} \sum_{i=1}^{n_{\text {typ }}} \rho_{i} \sum_{j=1}^{n_{\text {typ }}} \rho_{j} & {\left[\int_{r_{\text {in }}}^{r_{\text {out }}} r \frac{\mathrm{d}}{\mathrm{d} r}\left(\phi_{i j}(r)(1-S(r))\right) 4 \pi r^{2} g_{i j}(r) \mathrm{d} r\right.} \\
& \left.+\int_{r_{\text {out }}}^{\infty} r \frac{\mathrm{d} \phi_{i j}(r)}{\mathrm{d} r} 4 \pi r^{2} g_{i j}(r) \mathrm{d} r\right]
\end{aligned}
$$

where $i$ and $j$ run over the atom types, $g_{i j}(r)$ is the corresponding pair correlation function, $\rho_{i}$ and $\rho_{j}$ are the number densities of each type, and $S(r)$ is the switching function that takes the potential to zero. This function must be continuous and take values $S\left(r_{i n}\right)=1$ and $S\left(r_{\text {out }}\right)=0$; its derivative must also be continuous and take values $S^{\prime}\left(r_{\text {in }}\right)=S^{\prime}\left(r_{\text {out }}\right)=0$. 
If we assume the pair correlation function $g_{i j}(r) \approx 1$ beyond $r=r_{\text {in }}$ (which is usually a good approximation for liquids at relatively large distances), the improper integral in the second term of Equation (1) can be evaluated analytically for simple (e.g. inverse-power) forms of the pair potential $\phi_{i j}(r)$. Using a sixth-power dispersion form $\phi_{i j}(r)=-C_{i j}^{6} r^{-6}$, the improper integral becomes $\int_{r_{\text {out }}}^{\infty} 24 \pi C_{i j}^{6} r^{-4} \mathrm{~d} r=8 \pi r_{\text {out }}^{-3}$ and we have:

$$
p_{\text {exact }}-p_{\text {cut }} \approx p_{\text {corr }}=\frac{1}{6} \sum_{i=1}^{n_{\text {typ }}} \rho_{i} \sum_{j=1}^{n_{\text {typ }}} \rho_{j} C_{i j}^{6}\left[\int_{r_{\text {in }}}^{r_{\text {out }}}-r \frac{\mathrm{d}}{\mathrm{d} r}\left(\frac{1-S(r)}{r^{6}}\right) 4 \pi r^{2} \mathrm{~d} r+8 \pi r_{\text {out }}^{-3}\right]
$$

Applying integration by parts to the remaining integral gives

$$
p_{\text {corr }}=\frac{1}{6} \sum_{i=1}^{n_{\text {typ }}} \rho_{i} \sum_{j=1}^{n_{\text {typ }}} \rho_{j} C_{i j}^{6}\left[-4 \pi r_{\text {out }}^{-3}+\int_{r_{\text {in }}}^{r_{\text {out }}}\left(\frac{1-S(r)}{r^{6}}\right) 12 \pi r^{2} \mathrm{~d} r+8 \pi r_{\text {out }}^{-3}\right]
$$

and simplifying and rearranging leaves us with

$$
\begin{aligned}
p_{\text {corr }} & =\frac{1}{6} \sum_{i=1}^{n_{\text {typ }}} \rho_{i} \sum_{j=1}^{n_{\text {typ }}} \rho_{j} C_{i j}^{6}\left[4 \pi r_{\text {in }}^{-3}-\int_{r_{\text {in }}}^{r_{\text {out }}} 12 \pi r^{-4} S(r) \mathrm{d} r\right] \\
& =\frac{2 \pi}{3} \sum_{i=1}^{n_{\text {typ }}} \rho_{i} \sum_{j=1}^{n_{\text {typ }}} \rho_{j} C_{i j}^{6}\left((1-\lambda) r_{\text {in }}^{-3}+\lambda r_{\text {out }}^{-3}\right)
\end{aligned}
$$

with

$$
\lambda=\frac{3 \int_{r_{\text {in }}}^{r_{\text {out }}} r^{-4} S(r) \mathrm{d} r}{r_{\text {in }}^{-3}-r_{\text {out }}^{-3}}
$$

This form isolates the problematic integral $\int_{r_{\text {in }}}^{r_{\text {out }}} r^{-4} S(r) \mathrm{d} r$ which, depending on the form of the switching function $S(r)$, may be complicated or impossible to do analytically. For practical simulations, however, $\lambda$ can simply be precomputed using any suitable numerical method for a given value of $r_{\text {in }}, r_{\text {out }}$, and $S(r)$; this value can be used throughout the simulation.

We can also see that Equation (4) takes the form of a linear interpolation between the tail correction with a cutoff at $r_{\text {in }}$ and the correction with a cutoff of $r_{\text {out }}$; if we choose an $S(r)$ whose values are bounded between 0 and 1 then $\lambda$ will likewise be bounded between 0 and 1 .

On closer inspection, however, we can see that the interpolation endpoints are not the values the tail correction would take with a sharp cutoff: If we start with Equation (2) and take $S(r)=1$ for $r<r_{\text {out }}$, we get instead: 


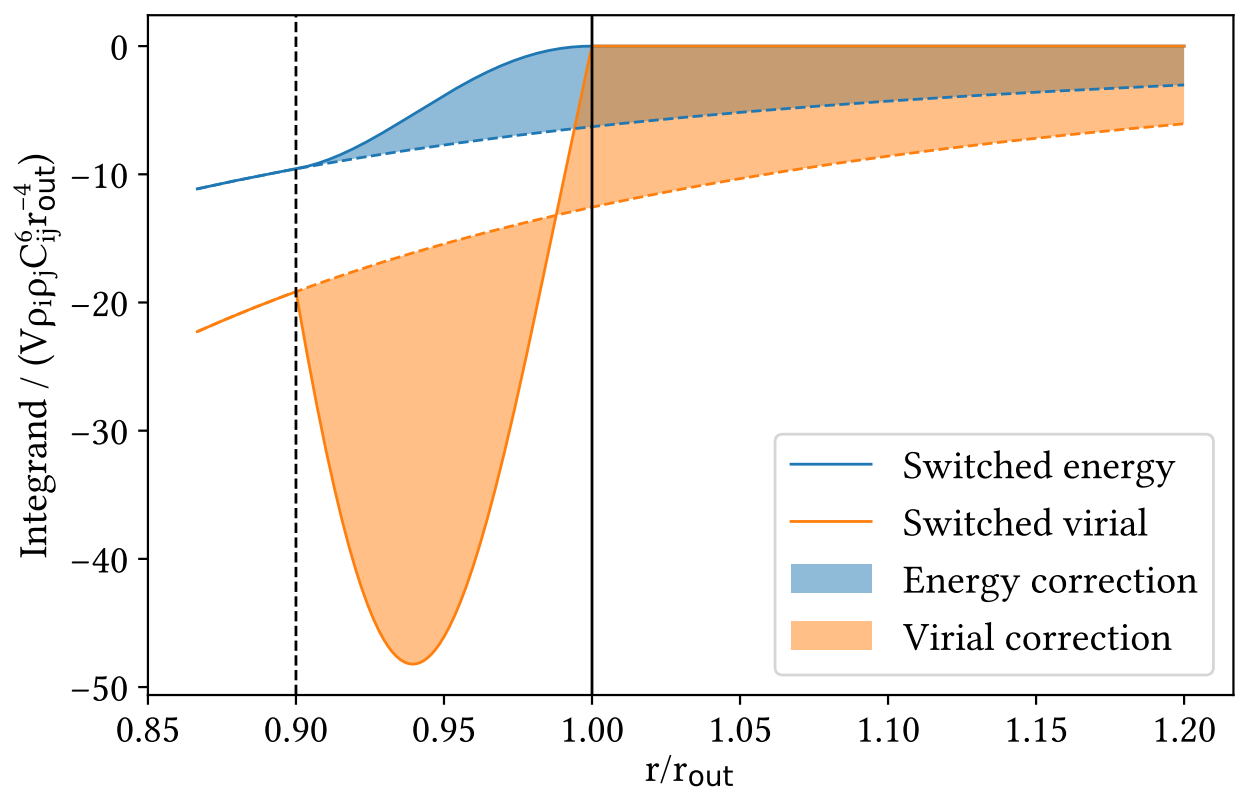

FIG. 10. Illustration of the integrals for the energy and virial tail corrections for a potential of the form $\phi_{\text {cut }}\left(r_{i j}\right)=C_{i j}^{6} r_{i j}^{-6} S(r)$. The dashed line corresponds to the potential with no cutoff $\left(f_{E}(r)=-\frac{1}{2} r^{-6} \cdot 4 \pi r^{2}\right.$ for the energy and $f_{W}(r)=\frac{1}{6} r \frac{\mathrm{d}}{\mathrm{d} r} r^{-6} \cdot 4 \pi r^{2}$ for the virial), while the solid line corresponds to the potential multiplied by the switching function $S(r)$. The shaded areas between the dashed and solid lines depict the integrals of Equations (2) (times $-V$ ) and (6); the area below the dotted line is negative. The energy and virial integrals (areas) are equal.

$$
p_{\text {exact }}-p_{\text {cut }, \text { sharp }} \approx \frac{4 \pi}{3} \sum_{i=1}^{n_{\text {typ }}} \rho_{i} \sum_{j=1}^{n_{\text {typ }}} \rho_{j} C_{i j}^{6} r_{\text {out }}^{-3},
$$

which is the correction Tildesley and Allen [45] give for the sixth-power part of an L-J potential, and twice the value we would get from Equation (4) by letting $\lambda=1$. This discrepancy is due to the extra $-4 \pi r_{\text {out }}^{-3}$ term that emerged from the integration by parts in Equation (3), and it can be physically interpreted as follows: With a sharp cutoff, an atom feels no force as it crosses the cutoff; the force just changes discontinuously from $-\phi^{\prime}\left(r \rightarrow r_{\text {out }}^{-}\right)$to zero. With a smooth cutoff, however, the switching function provides an extra gentle inward force as the atom exits the transition region. The extra virial due to this force provides an effective tail correction to the system's overall pressure, albeit only about half of the difference of the pressure with the sharp cutoff to the pressure of the ideal system with an infinite cutoff; Figure 10 provides an illustration of this idea. 
Tail corrections may also be computed, using the same method as above, for the energy. Although they do not affect the simulation dynamics in any way, they may be used for accurate bookkeeping and later analysis. The expression is [45, 46]

$$
\begin{aligned}
E_{\text {exact }}-E_{\text {cut }} & =\frac{V}{2} \sum_{i=1}^{n_{\text {typ }}} \rho_{i} \sum_{j=1}^{n_{\text {typ }}} \rho_{j} \int_{r_{\text {in }}}^{\infty}\left(\phi_{i j}(r)-\phi_{i j, \text { cut }}(r)\right) 4 \pi r^{2} g_{i j}(r) \mathrm{d} r \\
& \approx \frac{V}{2} \sum_{i=1}^{n_{\text {typ }}} \rho_{i} \sum_{j=1}^{n_{\text {typ }}} \rho_{j} C_{i j}^{6}\left[\int_{r_{\text {in }}}^{r_{\text {out }}}-(1-S(r)) 4 \pi r^{-4} \mathrm{~d} r+\int_{r_{\text {out }}}^{\infty}-4 \pi r^{-4} \mathrm{~d} r\right] \\
& =\frac{V}{2} \sum_{i=1}^{n_{\text {typ }}} \rho_{i} \sum_{j=1}^{n_{\text {typ }}} \rho_{j} C_{i j}^{6}\left[-\frac{4 \pi}{3} r_{\text {in }}^{-3}+\int_{r_{\text {in }}}^{r_{\text {out }}} 4 \pi r^{-4} S(r) \mathrm{d} r\right] \\
& =-\frac{2 \pi V}{3} \sum_{i=1}^{n_{\text {typ }}} \rho_{i} \sum_{j=1}^{n_{\text {typ }}} \rho_{j} C_{i j}^{6}\left((1-\lambda) r_{\text {in }}^{-3}+\lambda r_{\text {out }}^{-3}\right)
\end{aligned}
$$

with $\lambda$ defined as before. This is - coincidentally, with the $r^{-6}$ potential - identical to the virial correction, i.e. the pressure correction in Equation (4) multiplied by $-V$.

[1] M. J. Gillan, D. Alfé, A. P. Bartók, and G. Csányi, J. Chem. Phys. 139, 244504 (2013), arXiv:1303.0751v1.

[2] T. H. Dunning, J. Chem. Phys. 90, 1007 (1989).

[3] R. A. Kendall, T. H. Dunning, and R. J. Harrison, J. Chem. Phys. 96, 6796 (1992).

[4] T. B. Adler, G. Knizia, and H.-J. Werner, J. Chem. Phys. 127, 221106 (2007); G. Knizia, T. B. Adler, and H.-J. Werner, J. Chem. Phys. 130, 054104 (2009).

[5] L. Kong, F. A. Bischoff, and E. F. Valeev, Chem. Rev. 112, 75 (2012).

[6] S. Boys and F. Bernardi, Mol. Phys. 19, 553 (1970).

[7] H.-J. Werner, P. J. Knowles, G. Knizia, F. R. Manby, M. Schütz, P. Celani, T. Korona, R. Lindh, A. Mitrushenkov, G. Rauhut, K. R. Shamasundar, T. B. Adler, R. D. Amos, A. Bernhardsson, A. Berning, D. L. Cooper, M. J. O. Deegan, A. J. Dobbyn, F. Eckert, E. Goll, C. Hampel, A. Hesselmann, G. Hetzer, T. Hrenar, G. Jansen, C. Köppl, Y. Liu, A. W. Lloyd, R. A. Mata, A. J. May, S. J. McNicholas, W. Meyer, M. E. Mura, A. Nicklass, D. P. O’Neill, P. Palmieri, D. Peng, K. Pflüger, R. Pitzer, M. Reiher, T. Shiozaki, H. Stoll, A. J. Stone, R. Tarroni, T. Thorsteinsson, and M. Wang, "MOLPRO, version 2012.1, a pack- 
age of ab initio programs," (2012); H.-J. Werner, P. J. Knowles, G. Knizia, F. R. Manby, and M. Schütz, WIREs Comput Mol Sci 2, 242 (2012); M. Schütz, R. Lindh, and H.-J. Werner, Mol. Phys. 96, 719 (1999); R. Lindh, Theor. Chim. Acta 85, 423 (1993).

[8] A. Hjorth Larsen, J. Jørgen Mortensen, J. Blomqvist, I. E. Castelli, R. Christensen, M. Dułak, J. Friis, M. N. Groves, B. Hammer, C. Hargus, E. D. Hermes, P. C. Jennings, P. Bjerre Jensen, J. Kermode, J. R. Kitchin, E. Leonhard Kolsbjerg, J. Kubal, K. Kaasbjerg, S. Lysgaard, J. Bergmann Maronsson, T. Maxson, T. Olsen, L. Pastewka, A. Peterson, C. Rostgaard, J. Schiøtz, O. Schütt, M. Strange, K. S. Thygesen, T. Vegge, L. Vilhelmsen, M. Walter, Z. Zeng, and K. W. Jacobsen, J. Phys. Condens. Matter 29, 273002 (2017).

[9] S.-W. Chao, A. H.-T. Li, and S. D. Chao, J. Comput. Chem. 30, 1839 (2009).

[10] R. Hellmann, E. Bich, and E. Vogel, J. Chem. Phys. 128, 214303 (2008).

[11] A. H.-T. Li and S. D. Chao, J. Chinese Chem. Soc. 63, 282 (2016).

[12] W. L. Jorgensen, D. S. Maxwell, and J. Tirado-Rives, J. Am. Chem. Soc. 118, 11225 (1996).

[13] S. Plimpton, J. Comput. Phys. 117, 1 (1995).

[14] Using LAMMPS stable release from 5 Oct 2015.

[15] A. Brünger, C. L. Brooks, and M. Karplus, Chem. Phys. Lett. 105, 495 (1984).

[16] S. Nosé, J. Chem. Phys. 81, 511 (1984).

[17] W. G. Hoover, Phys. Rev. A 31, 1695 (1985).

[18] M. Parrinello and A. Rahman, J. Appl. Phys. 52, 7182 (1981).

[19] W. Shinoda, M. Shiga, and M. Mikami, Phys. Rev. B 69, 134103 (2004).

[20] M. E. Tuckerman, J. Alejandre, R. López-Rendón, A. L. Jochim, and G. J. Martyna, J. Phys. A. Math. Gen. 39, 5629 (2006).

[21] A. P. Bartók, M. J. Gillan, F. R. Manby, and G. Csányi, Phys. Rev. B - Condens. Matter Mater. Phys. 88, 054104 (2013).

[22] A. P. Bartók and G. Csányi, Int. J. Quantum Chem. 115, 1051 (2015).

[23] A. Bartók-Pártay, L. Bartók-Pártay, F. Bianchini, A. Butenuth, M. Caccin, S. Cereda, G. Csányi, A. Comisso, T. Daff, S. John, C. Gattinoni, G. Moras, J. Kermode, L. Mones, A. Nichol, D. Packwood, L. Pastewka, G. Peralta, I. Solt, O. Strickson, W. Szlachta, C. Varnai, M. Veit, and S. Winfield, "libAtoms+QUIP," (2018), http://libatoms.org.

[24] https://doi.org/10.17863/CAM. 26364.

[25] http://www.libatoms.org/Home/DataRepository. 
[26] W. L. Jorgensen and J. Tirado-Rives, J. Am. Chem. Soc. 110, 1657 (1988).

[27] W. D. Cornell, P. Cieplak, C. I. Bayly, I. R. Gould, K. M. Merz, D. M. Ferguson, D. C. Spellmeyer, T. Fox, J. W. Caldwell, and P. A. Kollman, J. Am. Chem. Soc. 117, 5179 (1995).

[28] S. J. Clark, M. D. Segall, C. J. Pickard, P. J. Hasnip, M. J. Probert, K. Refson, and M. C. Payne, Z. Krist. 220, 567 (2005).

[29] R. M. Parrish, L. A. Burns, D. G. A. Smith, A. C. Simmonett, A. E. DePrince, E. G. Hohenstein, U. Bozkaya, A. Y. Sokolov, R. Di Remigio, R. M. Richard, J. F. Gonthier, A. M. James, H. R. McAlexander, A. Kumar, M. Saitow, X. Wang, B. P. Pritchard, P. Verma, H. F. Schaefer, K. Patkowski, R. A. King, E. F. Valeev, F. A. Evangelista, J. M. Turney, T. D. Crawford, and C. D. Sherrill, J. Chem. Theory Comput. 13, 3185 (2017).

[30] T. Verstraelen, P. Tecmer, F. Heidar-Zadeh, C. E. González-Espinoza, M. Chan, T. D. Kim, K. Boguslawski, S. Fias, S. Vandenbrande, D. Berrocal, and P. W. Ayers, "HORTON," (2017), http://theochem.github.com/horton/.

[31] A. D. Becke, J. Chem. Phys. 88, 2547 (1988).

[32] A. D. Becke and R. M. Dickson, J. Chem. Phys. 89, 2993 (1988).

[33] V. Lebedev and D. Laikov, Dokl. Math. 59, 477 (1999).

[34] M. Ceriotti, J. More, and D. E. Manolopoulos, Comput. Phys. Commun. 185, 1019 (2014).

[35] Using LAMMPS stable release from 11 Aug 2017.

[36] A. Jones and B. Leimkuhler, J. Chem. Phys. 135, 84125 (2011).

[37] D. Quigley and M. I. J. Probert, J. Chem. Phys. 120, 11432 (2004), arXiv:0403756 [cond-mat].

[38] L. Martínez, R. Andrade, E. G. Birgin, and J. M. Martínez, J. Comput. Chem. 30, 2157 (2009).

[39] M. Ceriotti, G. Bussi, and M. Parrinello, J. Chem. Theory Comput. 6, 1170 (2010), arXiv:1204.0822v1.

[40] M. Ceriotti, G. Bussi, and M. Parrinello, Phys. Rev. Lett. 103, 30603 (2009).

[41] M. Ceriotti and D. E. Manolopoulos, Phys. Rev. Lett. 109, 100604 (2012).

[42] M. Ceriotti, G. Bussi, and M. Parrinello, Phys. Rev. Lett. 102, 020601 (2009), arXiv:0812.1168.

[43] A. Sokal, in Functional Integration. NATO ASI Series (Series B Physics), edited by C. DeWitt-Morette, P. Cartier, and A. Folacci (Springer, Boston, MA, 1997) pp. 131-192.

[44] G. J. Martyna, D. J. Tobias, and M. L. Klein, J. Chem. Phys. 101, 4177 (1994). 
[45] M. P. Allen and D. J. Tildesley, Computer simulation of liquids (Clarendon Press, Oxford, 1989).

[46] H. Sun, J. Phys. Chem. B 102, 7338 (1998).

[47] R. W. Hockney and J. W. Eastwood, Computer simulation using particles (Hilger, Bristol, 1988). 
